\title{
Le confort thermique en climat tempéré $\left({ }^{*}\right)$
}

\author{
J.-C. Deval \\ Laboratoire d'Ecothermique Solaire, CNRS, B.P. 21, 06562 Valbonne Cedex, France
}

(Reçu le 17 octobre 1983, révisé le 19 mars 1984, accepté le 23 mars 1984)

Résumé. - Le confort thermique de l'être humain en climat tempéré résulte du bilan des divers échanges avec l'environnement.

Cet article s'appuie sur des travaux effectués les 45 dernières années, et plus particulièrement des travaux récents dans le domaine de la physiologie de la thermorégulation.

Un modèle est construit à partir duquel sont établis des diagrammes pour les situations de confort.

\begin{abstract}
A better understanding of physiological phenoma is essential in designing buildings for human comfort.
This paper reviews part of the research of the last 45 years in physiology and bioengineering.

A comprehensive mathematical model of thermal comfort is described and comfort diagrams are given as tools for designers.
\end{abstract}

Nomenclature des notations utilisées.

$A C T$ : Activité du sujet

$A_{\text {DU }} \quad$ : Surface de Du Bois

$A_{\text {EFF }}$ : Surface effectivement rayonnante

$C_{\mathrm{RES}} \quad$ : Echanges par convection respiratoire (respiration sèche)

DH : Variation d'enthalpie

DT : Ecart de température

$\varepsilon_{\mathrm{clo}} \quad$ : Emissivité des vêtements

$E_{\text {COND }}:$ Echanges par conduction

$E_{\mathrm{CONV}}$ : Echanges par convection

$E_{\mathrm{DIF}}:$ Echanges par diffusion de la vapeur d'eau

$E_{\mathrm{RAD}}$ : Echanges par rayonnement

$E_{\mathrm{RES}}$ : Echanges par évaporation respiratoire

$E_{\mathrm{SW}} \quad$ : Echanges par évaporation de la sueur

$F A C L$ : Rapport de la surface du corps habillé sur la surface du corps nu

$F_{\text {EFF }} \quad$ : Rapport de la surface effectivement rayonnante du corps habillé sur la surface du corps habillé

(*) Ce travail a été présenté en partie lors de la rencontre 1983 de la Société Française des Thermiciens.

$\left(\mathrm{m}^{2}\right)$

(W)

(kJ/mole)

(K)

(一)

(W)

(W)

(W) $h \quad:$ Taille de l'individu

$h_{\mathrm{C}} \quad$ : Coefficient de transfert convectif

$\left(\mathrm{m}^{2}\right) \quad h_{\mathrm{Cc}} \quad:$ Coefficient de transfert convec-

$h_{\mathrm{Cm}}$

: Coefficient de transfert convectif au niveau des mains

$h_{\mathrm{Ct}}$ : Coefficient de transfert convectif au niveau de la tête

$h_{\mathrm{e}} \quad$ : Coefficient d'échange évaporatif

$h_{\mathrm{R}} \quad$ : Coefficient de transfert radiatif

$h_{\mathbf{R}_{\mathbf{c}}} \quad$ : Coefficient de transfert radiatif de la partie habillée du corps

$h_{\mathrm{Rm}} \quad$ : Coefficient de transfert radiatif au niveau des mains

$h_{\mathrm{Rt}} \quad$ : Coefficient de transfert radiatif au niveau de la tête

$H \quad$ : Production de chaleur interne (surfacique)

$m \quad$ : Mouillure de la peau

$M_{\text {B }} \quad$ : Métabolisme basal

$M_{\mathrm{T}} \quad$ : Métabolisme énergétique total mis en jeu par l'organisme

$\phi \quad$ : Degré hygrométrique de l'air

$\mathrm{Pa}$ : Pression partielle de vapeur d'eau dans l'air

$P_{\text {sat }} \quad$ : Pression de vapeur saturante

$Q \quad$ : Quotient respiratoire

$\mathcal{R}$ : Résistance thermique des vêtements (m)

$\left(\mathrm{W} / \mathrm{m}^{2} \cdot \mathrm{K}\right)$

$\left(\mathrm{W} / \mathrm{m}^{2} \cdot \mathrm{K}\right)$

$\left(\mathrm{W} / \mathrm{m}^{2} . \mathrm{K}\right)$

$\left(\mathrm{W} / \mathrm{m}^{2} \cdot \mathrm{K}\right)$

$\left(\mathrm{W} / \mathrm{m}^{2} \cdot \mathrm{mb}\right)$

$\left(W / m^{2} . K\right)$

$\left(W / m^{2} \cdot K\right)$

$\left(W / m^{2} \cdot K\right)$

$\left(\mathrm{W} / \mathrm{m}^{2} . \mathrm{K}\right)$

$\left(\mathrm{W} / \mathrm{m}^{2}\right)$

(一)

$\left(\mathrm{W} / \mathrm{m}^{2}\right)$

$\left(\mathrm{W} / \mathrm{m}^{2}\right)$

$(\%)$

(mm Hg)

(mm Hg)

(一)

$\left(\mathrm{m}^{2} . \mathrm{C} / \mathrm{W}\right)$ 
$\mathcal{R}_{\text {clo }}$ : Résistance thermique des vêtements

$r \quad$ : Rendement mécanique

$(-)$

$(-)$

$\left(\mathrm{W} / \mathrm{m}^{2}\right)$

$S \quad$ : Stockage dans l'organisme

$\sigma \quad$ : Constante de Boltzmann

$\mathrm{T}_{\mathrm{A}}$ : Température ambiante

$\mathrm{T}_{\text {air }}$ : Température de l'air

$\mathrm{T}_{\text {clo }}:$ Température de surface des vêtements

$\mathrm{T}_{\text {exp }} \quad$ : Température de l'air expiré

$T_{\text {MR }}$ : Température moyenne radiante

( $\left.{ }^{\circ} \mathrm{C}\right)$

$\mathrm{T}_{\text {sk }} \quad$ : Température moyenne cutanée

: Humidité relative de la peau

$(\mathrm{m} / \mathrm{s})$

(kg)

$(\mathrm{g} / \mathrm{kg})$

$\left(\mathrm{W} / \mathrm{m}^{2}\right)$

\section{Introduction.}

Les études concernant les économies d'énergies dans l'habitat conduisent indubitablement à se préoccuper du confort thermique de l'être humain. Pourtant, il semble que pour beaucoup de concepteurs, la qualité radiative des ambiances soit ignorée et qu'une valeur de $19^{\circ}$ pour la température d'air soit la seule préoccupation de leurs programmes. Des recherches sur les ambiances radiatives et des expériences s'y rapportant ont pourtant été faites afin de montrer que le confort pouvait être obtenu diversement et éventuellement plus économiquement qu'en se limitant à imposer une valeur pour la température d'air.

Les physiologues n'ont pas attendu ces dernières années pour s'intéresser à la machine thermique humaine, mais l'aspect médical est celui qui était principalement retenu. Les recherches effectuées l'ont souvent été dans des conditions particulières d'ambiances pour des raisons de connaissance des limites de résistance de l'être humain amené à faire des travaux dans ces ambiances. Aussi convient-il de regrouper ces recherches en tenant compte de leurs spécificités afin d'établir l'ensemble des conditions qui sont associées à des situations de confort.

C'est l'objet du travail ci-après qui, sous un aspect " thermique » résume l'analyse critique de nombreux travaux et actualise ainsi les travaux de P. O. Fanger. Il aboutit à des équations de confort et à des diagrammes destinés aux concepteurs d'habitats dits " économes » en énergie.

La complexité de la machine humaine, qui fait appel à des processus d'échanges divers, des systèmes de protection et de défense, est telle qu'il a fallu séparer ce sujet en deux et ne traiter ici que des climats tem-

pérés. Le cas des climats chauds, très différent quant aux réactions de l'être humain, fera l'objet d'une autre publication.

\section{Bilan énergétique de l'homme dans ses échanges avec son environnement.}

2.1 LE CONFORT THERMIQUE. - Le concept de confort thermique peut être caractérisé, pour un individu donné, par l'état de satisfaction avec les conditions d'environnement thermique [1]. Cette satisfaction est traduite par l'impossibilité pour le sujet de préciser s'il préfère un environnement plus « froid " ou plus " chaud ». Bien sûr, les gens sont différents, biologiquement et psychiquement, et si des individus se trouvent soumis aux mêmes conditions thermiques, dans une même pièce, il n'est normalement pas possible de les satisfaire tous en même temps. Toutefois, on peut créer un climat optimal dans une pièce, c'est-àdire des conditions dans lesquelles le pourcentage maximal d'individus est dans un état de confort thermique.

Les paramètres les plus importants qui participent au bilan thermique de l'homme et caractérisent l'état de confort thermique sont :

- le niveau d'activité du sujet (correspondant à une production interne de chaleur dans le corps).

- la résistance thermique des vêtements,

- la température de l'air,

- la vitesse relative de l'air,

- la température moyenne radiante,

- le degré hygrométrique de l'air.

2. 2 LE BILAN THERMiQUe. - Globalement, on peut écrire le bilan énergétique entre l'homme et son environnement de la manière suivante, en comptabilisant les contributions de la production interne de chaleur, des échanges rayonnants, convectifs, conductifs, évaporatoires et respiratoires.

$$
\begin{array}{r}
S=H+E_{\mathrm{RAD}}+E_{\mathrm{CONV}}+E_{\mathrm{COND}}+E_{\mathrm{SW}}+ \\
+E_{\mathrm{DIF}}+E_{\mathrm{RES}}+C_{\mathrm{RES}} .
\end{array}
$$

L'homme est un être homéotherme et dispose d'une stratégie métabolique efficace lui permettant de déclencher tout un processus de régulation qui facilite son adaptation au milieu.

Hypothèse : Pour que l'homme soit dans un état de confort thermique, il faut que le débit de stockage dans l'organisme soit nul : autrement dit, qu'il puisse évacuer l'énergie produite par son activité métabolique.

$$
S=0 \text {. }
$$

C'est l'assertion qui a permis à P. O. Fanger dès 1967 [2] d'élaborer sa fameuse équation de confort. 
Cette hypothèse ne fait pas état des processus transitoires. Dans cette étude on traitera le cas d'un individu en équilibre thermique avec son environnement. Cela impose un régime établi et un sujet acclimaté, non en situation d'adaptation ou de défense à des contraintes thermiques.

2.3 LE MÉTABOLISME THERMIQUE. - La vie de l'être humain est assurée par diverses réactions énergétiques qui évacuent l'énergie emmagasinée dans les cellules. Si le comburant est l'oxygène que nous respirons, l'un des produits formés est l'eau qu'il convient d'éliminer.

Le métabolisme énergétique de l'être humain provient principalement de la réaction d'oxydation du glucose.

$$
\mathrm{C}_{6} \mathrm{H}_{12} \mathrm{O}_{6}+6 \mathrm{O}_{2} \rightarrow 6 \mathrm{CO}_{2}+6 \mathrm{H}_{2} \mathrm{O}
$$

avec une variation d'enthalpie :

$$
D H=-2813 \mathrm{~kJ} / \text { mole } \text {. }
$$

L'oxydation d'une molécule de glucose conduit à la formation de 38 molécules d'ATP et c'est lors de la réaction d'hydrolyse de la molécule d'ATP que l'ADN se forme.
Il apparaît clairement que la mesure de la consommation d'oxygène permet d'évaluer la quantité de chaleur métabolique $M_{\mathrm{T}}$, c'est-à-dire le métabolisme énergétique total.

La relation (5) donne l'expression du métabolisme (en watts par mètre carré de surface de peau) en fonction de la consommation d'oxygène $V_{\mathrm{O}_{2}}$ (en litres par minute) dans les conditions normales de température et de pression, et du quotient respiratoire $Q$ qui varie de 0,83 au repos à 1 lors d'un travail modéré [3].

$$
M_{\mathrm{T}}=352(0,23 Q+0,77)\left(V_{\mathrm{O}_{2}} / A_{\mathrm{DU}}\right),
$$

$A_{\text {DU }}$ est la surface du corps nu, dite aussi surface de «Du Bois » [4]

$$
A_{\text {DU }}=0,203 w^{0,425} h^{0,725} \text { en } \mathrm{m}^{2}
$$

où $w$ est le poids de l'individu en $\mathrm{kg}$,

$h$ est la taille de l'individu en $\mathrm{m}$.

Au repos, la totalité de l'énergie produite par l'homme se transforme en chaleur. C'est le métabolisme basal $M_{\mathrm{B}}$. La valeur généralement adoptée est de $58,15 \mathrm{~W} / \mathrm{m}^{2}$. Au cours d'un travail, une fraction de l'énergie fournit de l'énergie mécanique et n'est donc pas dégradée dans l'organisme sous forme de chaleur.

$$
H \quad=\quad M_{\mathrm{T}} \quad-W
$$

production de chaleur interne $=$ métabolisme énergétique total - travail ou métabolisme thermique mis en jeu par l'organisme

On peut introduire la notion de rendement mécanique :

$$
r=W / M_{\mathrm{T}} .
$$

Dans la majorité des cas, ce rendement est négligeable. Malgré tout pour certaines activités, il peut atteindre 0,2 à 0,25 .

L'unité adoptée le plus souvent est le «Met » où 1 Met correspond au métabolisme d'un individu assis au repos.

$$
1 \mathrm{Met}=58,15 \mathrm{~W} / \mathrm{m}^{2}
$$

ce qui permet de définir le paramètre «activité » $(A C T)$

$$
A C T=\frac{M_{\mathrm{T}}}{58,15} .
$$

Le tableau I donne le métabolisme énergétique total mis en jeu pour différentes activités.

2.4 LeS ÉCHANGeS DE CHALEUR RADiATIFs. L'échange radiatif de chaleur entre le corps humain et son environnement $[5-7,10]$ est souvent considéré comme un procédé dont il faut s'accommoder, qui est d'ailleurs fort mal pris en compte et dont on se préoc-
Tableau I. - Métabolisme énergétique total pour différentes activités.

$\begin{array}{lcc}\text { Sommeil } & 40 \mathrm{~W} / \mathrm{m}^{2} \text { soit } & 0,7 \mathrm{Met} \\ \text { Couché } & 45 & 0,8 \\ \text { Assis } & 60 & 1,0 \\ \text { Travail mental assis } & 65 & 1,1 \\ \text { Debout } & 70 & 1,2 \\ \text { Marche à } 4 \mathrm{~km} / \mathrm{h} & 130 & 2,2 \\ \text { Marche }(4 \mathrm{~km} / \mathrm{h}) \text { et charge de } & & \\ \quad 20 \mathrm{~kg} & 220 & 3,8 \\ \text { Course à pied à } 10 \mathrm{~km} / \mathrm{h} & 440 & 7,6 \\ \text { Course à pied à } 17,5 \mathrm{~km} / \mathrm{h} & 830 & 14,3 \\ \text { Course à pied à } 25 \mathrm{~km} / \mathrm{h} & 2500 & 43,0 \\ \text { Course à pied à } 30 \mathrm{~km} / \mathrm{h} & 6100 & 104,8 \\ \text { Nage à } 1,6 \mathrm{~km} / \mathrm{h} & 315 & 5,4 \\ \text { Nage à } 2,35 \mathrm{~km} / \mathrm{h} & 760 & 13,1 \\ \text { Football } & 600 & 10,3\end{array}$

cupe peu des possibilités qu'il offre. Les échanges radiatifs représentent pourtant environ la moitié du bilan du corps humain.

L'importance de la chaleur rayonnante est illustrée par le fait que l'on peut se déshabiller et prendre un bain de soleil en montagne alors que le thermomètre est en dessous de $0^{\circ} \mathrm{C}$. 


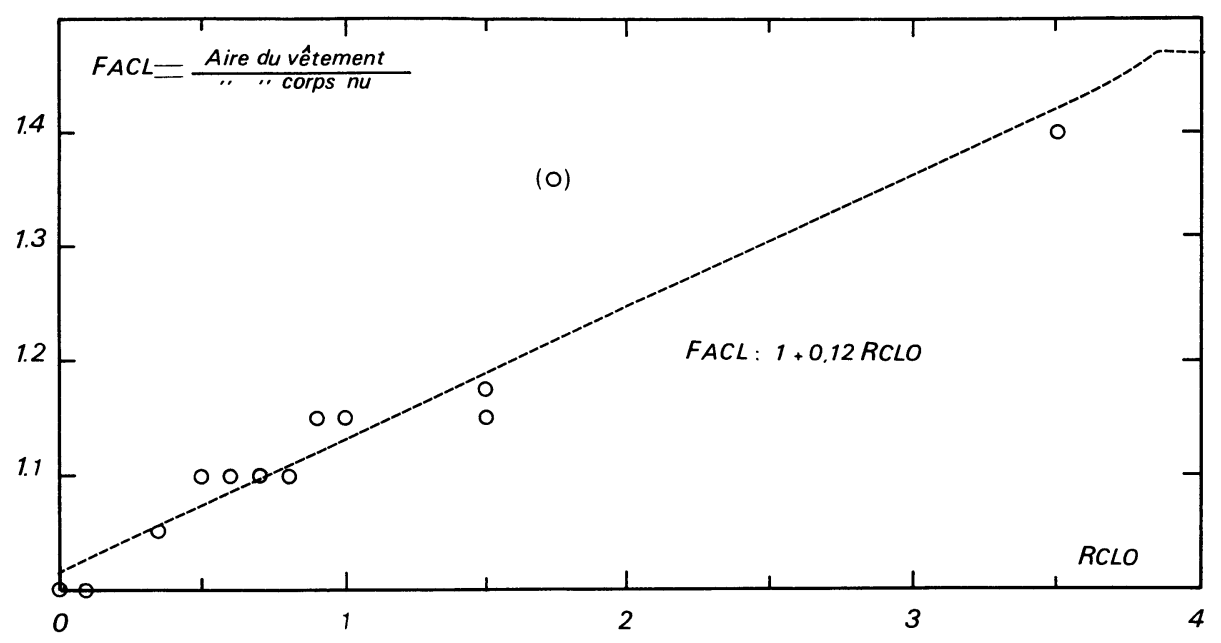

Fig. 1. - Rapport de la surface du corps habillé sur la surface du corps nu en fonction de la résistance thermique (en clo) des vêtements.

[The ratio of the surface area of the clothed body to the surface area of the nude body as a function of the thermal resistance from skin to outer surface of the clothed body (unit : clo).]

Pour écrire l'expression du flux radiatif, il faut tout d'abord définir la surface effectivement rayonnante $A_{\mathrm{EFF}}$ vers l'environnement.

$$
A_{\mathrm{EFF}}=A_{\mathrm{DU}} * F_{\mathrm{EFF}} .
$$

Elle est liée à la surface de Du Bois par un facteur postural $F_{\mathrm{EFF}}$ purement géométrique.

$$
\begin{aligned}
& F_{\text {EFF }}=0,696 \text { pour un sujet assis } \\
& F_{\text {EFF }}=0,725 \text { pour un sujet debout } .
\end{aligned}
$$

L'émissivité $\varepsilon_{\text {clo }}$ moyenne des vêtements portés sous nos latitudes est d'environ 0,95 . Pour les vêtements blancs portés dans le bassin méditerranéen (de type gandura ou cachabia par exemple) on pourra adopter $\varepsilon_{\text {clo }}=0,6$. On en déduit l'expression classique issue d'hypothèse de rayonnement de corps gris

$$
E_{\mathrm{RAD}}=A_{\mathrm{EFF}} \varepsilon_{\mathrm{clo}} F A C L \sigma\left(T_{\mathrm{clo}}^{4}-T_{\mathrm{MR}}^{4}\right)\left({ }^{1}\right) .
$$

A partir de la revue bibliographique établie par Fanger [19], on peut établir par une droite de régression (avec un coefficient de corrélation calculé de 0,93 ) que l'on a une augmentation de surface d'environ $12 \%$ par clo (Fig. 1),

$$
F A C L=1+0,12 R_{\text {clo }}
$$

l'expression (12) peut se linéariser sous la forme :

$$
\begin{array}{r}
E_{\mathrm{RAD}}=4 F_{\mathrm{EFF}} F A C L \varepsilon_{\mathrm{clo}} \sigma A_{\mathrm{DU}}\left(\left(T_{\mathrm{clo}}+T_{\mathrm{MR}}\right) / 2\right)^{3} \times \\
\times\left(T_{\mathrm{MR}}-T_{\mathrm{clo}}\right) .
\end{array}
$$

( $\left.{ }^{1}\right)$ Où $T_{\text {clo }}$ est la température moyenne de surface des vêtements et $T_{\mathrm{MR}}$ la température moyenne de rayonnement de l'environnement.
Cette première approximation traite le cas d'un sujet recouvert uniformément d'un vêtement fictif d'isolation moyenne $\mathcal{R}_{\text {clo }}$. Dans le modèle considéré ici le corps humain est décomposé en trois parties qui participent différemment aux échanges : la partie du corps habillée, la tête et les mains.

Chaque terme de l'équation est pondéré par les rapports surfaciques de ces parties du corps (respectivement $94 \%, 2 \%$ et $4 \%$ ).

La température de la tête est prise égale à la température moyenne cutanée $T_{\mathrm{sk}}$ alors que la température des mains est considérée plus basse de $6^{\circ} \mathrm{C}$ [8].

Finalement, on obtient l'expression suivante pour l'échange radiatif :

$$
\begin{aligned}
E_{\mathrm{RAD}}=\{ & h_{\mathrm{Rc}} F A C L\left(T_{\mathrm{MR}}-T_{\mathrm{clo}}\right) 0,94+ \\
& +h_{\mathrm{Rt}}\left(T_{\mathrm{MR}}-T_{\mathrm{sk}}\right) 0,02 \\
& \left.+h_{\mathrm{Rm}}\left(T_{\mathrm{MR}}-T_{\mathrm{sk}}-6\right) 0,04\right\} A_{\mathrm{EFF}}
\end{aligned}
$$

avec :

$$
\begin{aligned}
& h_{\mathrm{Rc}}=4 \varepsilon_{\mathrm{clo}} \sigma\left\{\left(T_{\mathrm{clo}}+T_{\mathrm{MR}}\right) / 2+273\right\}^{3} \\
& h_{\mathrm{Rt}}=4 \varepsilon_{\mathrm{sk}} \sigma\left\{\left(T_{\mathrm{sk}}+T_{\mathrm{MR}}\right) / 2+273\right\}^{3} \\
& h_{\mathrm{Rm}}=4 \varepsilon_{\mathrm{sk}} \sigma\left\{\left(T_{\mathrm{sk}}-6+T_{\mathrm{MR}}\right) / 2+273\right\}^{3} .
\end{aligned}
$$

Les indices $\mathrm{c}, \mathrm{t}$ et $\mathrm{m}$ correspondent respectivement au corps, à la tête et aux mains.

2. 5 Les ÉChANGeS De CHALEUR CONVeCtiFs. - Le corps humain échange de la chaleur avec le fluide qui l'environne (en général l'air) par convection [11-13]. La détermination des coefficients de convection $h_{\mathrm{C}}$ a été l'objet de nombreuses recherches ces dernières années. Les unes sont expérimentales, sur mannequin ou sur sujet vivant, les autres sont des modélisations théoriques qui simulent le corps humain par un ou plusieurs cylindres verticaux. On observe (Tableau II) 
Tableau II. $-h$ convectif en $\mathrm{W} / \mathrm{m}^{2}$.C.

\begin{tabular}{lccc}
\multicolumn{1}{c}{ Auteurs } & Année & $\begin{array}{c}\text { Convection } \\
\text { naturelle }\end{array}$ & $\begin{array}{c}\text { Convection forcée } \\
(0,2 \mathrm{~m} / \mathrm{s})\end{array}$ \\
Buettner & - & - & - \\
Winslow et al. & 1934 & 2,46 & 3,27 \\
Nelson et al. & 1939 & - & 4,65 \\
Winslow et al. & 1949 & - & 3,89 \\
Colin et al. & 1967 & 2,67 & 5,22 \\
Nishi et al. & 1970 & - & 5,63 \\
Rapp & 1973 & 4,0 & $3,10-4,10$ \\
Missenard & 1974 & - & - \\
Nishi et al. & 1977 & 3,0 & 5,17 \\
Valeur moyenne & & 3,12 & - \\
Ecart type & & 0,62 & 4,49 \\
& & & 0,91
\end{tabular}

une dispersion importante selon les auteurs pour le coefficient $h_{\mathrm{C}}$.

La formule la plus couramment utilisée en convection naturelle a été proposée par Nielsen et al. en 1952. C'est celle qui a été adoptée pour l'élaboration de la norme expérimentale X 35-204. C'est celle-ci que nous utiliserons.

$$
h_{\mathrm{C}}=2,38\left(T_{\mathrm{clo}}-T_{\text {air }}\right)^{0,25} .
$$

On peut noter que dans la norme apparaît la température moyenne cutanée au lieu de la température des vêtements. C'est la température de surface des vêtements notée $T_{\text {clo }}$ qui participe à l'échange (sauf bien entendu dans le cas d'un sujet nu).

Cette température sera évaluée plus loin (3.2.1).

Dans le cas des climats chauds où l'être humain porte des vêtements amples, on ne pourra plus considérer la température de surface des habits car le vêtement n'a alors plus une action d'isolation. L'existence d'une couche d'air entre le corps et le vêtement et l'existence d'un effet dit "soufflet" qui renouvelle l'air contraignent à déterminer différemment $h_{\mathrm{C}}$. Ce cas ne sera pas considéré ici. En convection forcée, $h_{\mathrm{C}}$ dépend de la vitesse relative de l'air $V_{\text {air }}$.

Winslow et al. proposent :

$$
h_{\mathrm{C}}=12,1 V_{\mathrm{air}}^{0,5} \text {. }
$$

Cette formule donne $5,4 \mathrm{~W} / \mathrm{m}^{2} .{ }^{\circ} \mathrm{C}$ pour une vitesse d'air de $0,2 \mathrm{~m} / \mathrm{s}$ et semble élevée par rapport aux résultats du tableau II. La valeur moyenne $\left(4,49 \mathrm{~W} / \mathrm{m}^{2} .{ }^{\circ} \mathrm{C}\right)$ permet d'ajuster la formule (20) et de proposer :

$$
h_{\mathrm{C}}=10 V_{\mathrm{air}}^{0,5} \text {. }
$$

C'est celle que nous adopterons bien qu'elle diffère de celle qui apparaît dans la norme expérimentale.

Tout comme pour l'évaluation des échanges radiatifs, le corps humain est divisé en trois parties (tête nue, mains nues et la partie habillée).
Finalement, on écrira :

$$
\begin{aligned}
& E_{\mathrm{CONV}}=\left\{h_{\mathrm{Cc}} F A C L\left(T_{\mathrm{air}}-T_{\mathrm{clo}}\right) 0,94+\right. \\
& +h_{\mathrm{Ct}}\left(T_{\mathrm{air}}-T_{\mathrm{sk}}\right) 0,02 \\
& \left.+h_{\mathrm{Cm}}\left(T_{\text {air }}-T_{\text {sk }}-6\right) 0,04\right\} A_{\mathrm{DU}} \text {, } \\
& h_{\mathrm{Cc}}=\operatorname{MAX}\left(2,38\left(T_{\text {clo }}-T_{\text {air }}\right)^{0.25}, 10 V_{\text {air }}^{0,5}\right) \\
& h_{\mathrm{Ct}}=\operatorname{MAX}\left(2,38\left(T_{\mathrm{sk}}-T_{\mathrm{air}}\right)^{0,25}, 10 V_{\mathrm{air}}^{0,5}\right) \\
& h_{\mathrm{Cm}}=\operatorname{MAX}\left(2,38\left(T_{\mathrm{sk}}-6-T_{\mathrm{air}}\right)^{0,25}, 10 V_{\mathrm{air}}^{0,5}\right) .
\end{aligned}
$$

\subsection{Les ÉCHANGeS DE CHALEUR PAR CONDUCTION. -} On peut s'affranchir dans la majeure partie des cas étudiés dans le domaine du confort thermique, des échanges conductifs. Le corps humain est en contact étroit avec un corps solide dans des circonstances particulières et pour que le flux devienne sensible, il faut que la surface en contact soit importante. A ce sujet, un cas intéressant est celui du repos allongé. L'isolation thermique d'un corps humain nu, allongé sans couverture sur un lit, ne correspond pas à un clo nul (voir Tableau IV) comme on pourrait le croire mais à 0,4 clo, ce qui n'est pas négligeable (communication personnelle de $\mathrm{P}$. O. Fanger).

Dans la vie courante, les contacts se font généralement avec des corps isolants (fauteuils, lits...), et ceci peut être considéré comme une isolation supplémentaire. Les contacts avec les corps effusifs ne correspondent qu'à des cas très particuliers, la plupart du temps évités, et qui ne se font que par des surfaces réduites. Ils ne seront pas considérés ici.

\subsection{Les ÉChaNges DE Chaleur PAR ÉVAPORATION} CUTANÉE. - La transpiration, définie comme étant l'excrétion de la sueur par les glandes sudoripares de la peau, est le processus de régulation thermique le plus important chez l'homme. La perte de chaleur par évaporation d'eau permet de réaliser, dans la mesure du possible, l'homéothermie du corps humain. L'évaporation de la sueur sécrétée peut atteindre pour l'homme non acclimaté à l'ambiance des valeurs de l'ordre de $500 \mathrm{~g} / \mathrm{h}$, ce qui donne, pour une chaleur latente de $2,45 \times 10^{6} \mathrm{~J} / \mathrm{kg}$, une déperdition de l'ordre de $350 \mathrm{~W}$.

Chez un sujet acclimaté, on peut atteindre des valeurs doubles [14].

L'autre mode de transfert cutané de masse et de chaleur est la perspiration insensible qui est une diffusion passive d'eau entre les couches cutanées et l'ambiance. Le flux est directement proportionnel à la différence de pression partielle de la vapeur d'eau dans l'air et la pression de saturation au niveau de la peau :

$$
E_{\mathrm{DIF}}=0,41 A_{\mathrm{DU}}\left(P_{\mathrm{sat}}\left(T_{\mathrm{sk}}\right)-P a\right) .
$$


Cette modalité correspond à des flux nettement plus faibles que l'évaporation de la sueur. Au repos et à la neutralité thermique $\left(T_{\text {air }}=T_{\mathrm{MR}}=28^{\circ} \mathrm{C}, \quad \mathrm{Pa}=\right.$ $20 \mathrm{mb}$ ), Willy et Newburgh (1931) ont déterminé une perspiration insensible de $11 \mathrm{~g} / \mathrm{h} \cdot \mathrm{m}^{2}$.

La transpiration se caractérise par un débit sudoral excrété par chaque pore. En fonction du débit (qui dépend des contraintes thermiques extérieures, de la température de la peau et du métabolisme), une partie importante est évaporée et l'autre ruisselle. Le ruissellement se produit quand une zone cutanée est sous la dépendance de deux glandes sudoripares, c'est-àdire quand les gouttes de sueur non évaporées se rejoignent. La limite est fonction de la nature de la peau mais aussi de la moiteur de l'air (combinaison de la température de l'air, son hygrométrie, et de la ventilation) qui facilite cette jonction de goutte, diminue les effets de capillarité et de tension de surface et conditionne la saturation de la sudation.

J. J. Vogt et son équipe du Centre Bioclimatique de Strasbourg ont montré qu'à partir de ce stade, le rendement évaporatoire $r_{\mathrm{e}}$ chute fortement :

$$
r_{\mathrm{e}}=\frac{\text { Quantité d'eau évaporée }}{\text { Quantité d'eau sécrétée }} \text {. }
$$

Ce rendement est uniquement fonction de la mouillure de la peau $m$ (rapport de la surface mouillée sur la surface totale), et non du débit sudoral (Fig. 2)

$$
r_{\mathrm{e}}=1-0,42 \exp \{-6(1-m)\} .
$$

Ce phénomène est important car la sueur qui ruisselle et tombe au sol ne participe pas au refroidissement du corps. On admet qu'une mouillure de 0,3 est optimale (communication personnelle de J. J. Vogt).

Passons en revue les différentes études relatives aux échanges par évaporation cutanée :

En 1937, Gagge propose la formulation :

$$
E_{\mathrm{Sw}}=h_{\mathrm{e}} m\left(P_{\mathrm{sat}}\left(T_{\mathrm{sk}}\right)-P a\right) A_{\mathrm{DU}}
$$

où $m$ est la mouillure de la peau.

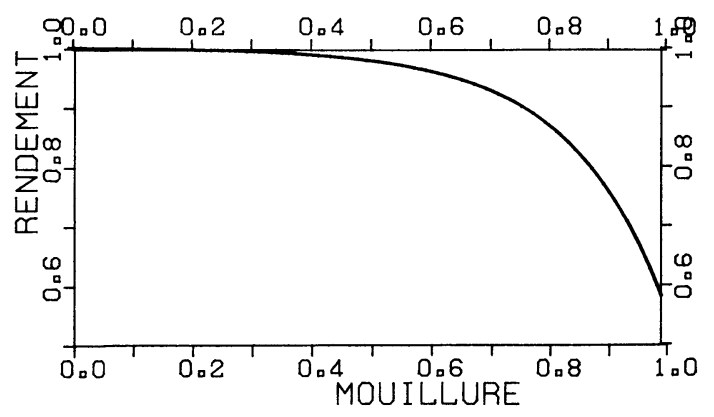

Fig. 2. - Rendement évaporatoire de la sudation thermique $\left(r_{\mathrm{e}}\right)$ en fonction de la mouillure cutanée $(m)$ (d'après Vogt).

[Relationship between evaporative sweating efficiency $\left(r_{\mathrm{e}}\right)$ and skin wettedness $(m)$.]
Buettner en 1938 introduit la notion d'humidité relative de la peau $\mu$,

$$
E_{\mathrm{Sw}}=h_{\mathrm{e}}\left(\mu P_{\mathrm{sat}}\left(T_{\mathrm{sk}}\right)-P a\right) A_{\mathrm{DU}} .
$$

Les deux notions sont, bien entendu, équivalentes en posant :

$$
m=\frac{\mu P_{\mathrm{sat}}\left(T_{\mathrm{sk}}\right)-P a}{P_{\mathrm{sat}}\left(T_{\mathrm{sk}}\right)-P a} .
$$

Le coefficient d'échange $h_{\mathrm{c}}$ a été évalué expérimentalement et différents auteurs proposent :

Lewis (1922)

$$
h_{\mathrm{e}}=2.2 h_{\mathrm{C}}
$$

où $h_{\mathrm{C}}$ est le coefficient de transfert par convection.

Nelson et al. (1947)

$$
h_{\mathrm{e}}=4,9
$$

Aikas et al. (1963)

$$
h_{\mathrm{e}}=5,25
$$

Rapp (1970)

$$
\begin{aligned}
& h_{\mathrm{e}}=8,5 V_{\mathrm{air}}^{0,25} \mathrm{en} \mathrm{convect.} \mathrm{forcée} \\
& h_{\mathrm{e}}=2,95 D T^{0,258} \text { en convect. libre }
\end{aligned}
$$

avec

$$
D T=\operatorname{ABS}\left(T_{\text {sk }}-T_{\text {air }}\right)
$$

Candas et al. (1979)

$$
\begin{array}{r}
h_{\mathrm{e}}=13 V_{\text {air }}^{0,4} \text { pour } 0,3<V_{\text {air }}<0,9 \\
(\mathrm{~m} / \mathrm{s})
\end{array}
$$

La théorie de la sudation requise [14-18] met en évidence la mouillure cutanée à réaliser et le débit sudoral correspondant pour assurer la neutralité thermique.

Cette technique semble plus adaptée à l'étude des ambiances chaudes qu'aux ambiances de confort dans lesquelles la sudation ne varie pas dans de larges marges.

Pour l'évaluation des pertes calorifiques par évaporation cutanée, nous avons choisi pour le modèle, la formule proposée par Fanger [5, 19] qui donne un flux optimal fonction de l'activité du sujet.

$$
E_{\mathrm{SW}}=0,49 A_{\underline{\mathrm{DU}}}\left(M_{\mathrm{B}}-M_{\mathrm{T}}\right)
$$

où $M_{\mathrm{B}}$ est le métabolisme basal $\left(\mathrm{W} / \mathrm{m}^{2}\right)$ et $M_{\mathrm{T}}$ le métabolisme total $\left(\mathrm{W} / \mathrm{m}^{2}\right)$. Cette formule empirique a été élaborée par Fanger à partir des résultats sur le nuage de points correspondant $\left(E_{\mathrm{SW}}=f\left(M_{\mathrm{T}}\right)\right)$.

Tout ceci n'est malheureusement exprimé que pour des conditions voisines de l'ambiance en pays tempéré.

Pour le cas des pays chauds, non considéré ici, il convient de modifier ces formules pour tenir compte des actions combinées température-hygrométrie-ventilation qui sont alors très importantes. 
2.8 LES ÉCHANGES RESPIRATOIRES. - L'air à la sortie des poumons est pratiquement saturé d'humidité. Il y a échange couplé de masse et de chaleur dans les poumons qui fonctionnent comme un échangeur de surface quasi infini (de l'ordre de $100 \mathrm{~m}^{2}$ ).

On peut séparer l'échange de chaleur en deux termes : l'un $E_{\mathrm{RES}}$ dû à la chaleur latente de la vapeur d'eau, l'autre $C_{\mathrm{RES}}$ dû à l'élévation de la température de l'air expiré.

Les deux termes sont bien entendu liés au métabolisme par l'intermédiaire de la ventilation des poumons en oxygène.

Fanger [19] donne les termes :

$$
E_{\mathrm{RES}}=0,0023 M_{\mathrm{T}}(44-P a)\left(\frac{4180}{3600}\right)
$$

où $P a$ est la pression partielle de vapeur d'eau dans l'air inspiré à la température de l'air ambiant $T_{\text {air }}$. $\mathrm{Pa}$ peut être obtenue à partir de l'humidité relative $\phi(\mathrm{en} \%)$ et de la pression de vapeur saturante de l'air

$$
P a=\frac{\phi}{100} \cdot P_{\text {sat }}\left(T_{\text {air }}\right),
$$

la pression de saturation peut être tirée des diagrammes de l'air humide ou obtenue de manière suffisamment précise dans la zone de température qui nous intéresse par une approximation par polynôme de Lagrange

$$
\begin{array}{r}
P_{\text {sat }}(T)=760 \times 6,05 \times 10^{-7}[((T+7,066) T+ \\
+908,88) T+9567] / 1,013 .
\end{array}
$$

McCutchan et Taylor [20] donnent l'expression de l'échange respiratoire par chaleur sensible.

$$
C_{\mathrm{RES}}=0,014 M_{\mathrm{T}}\left(T_{\exp }-T_{\mathrm{air}}\right)\left(\frac{4180}{3600}\right)
$$

où $T_{\text {exp }}$ est la température de l'air expiré.

$$
T_{\text {exp }}=32,6+0,066 T_{\text {air }}+32 \times 10^{-3} \mathrm{Wa}
$$

où $W a$ est le poids d'eau (en grammes) par kg d'air sec de l'air inspiré.

\section{Température de peau et température des vêtements.}

\subsection{LA TEMPÉRATURE CUTANÉE.}

3.1.1 Remarques. - La peau constitue l'interface biologique entre l'organisme et l'extérieur. Son état, caractérisé par la race, le sexe, l'âge ou l'adiposité, intervient dans l'appréciation de confort ressenti par l'être humain. C'est pourquoi il ne faut pas vouloir de rigueur dans les résultats avancés, et il ne peut être qu'indicatif de transposer des résultats obtenus sur des sujets d'Europe ou des Etats-Unis pour des climats tempérés à des peuples et à des climats très différents.

Par ailleurs, un bon nombre d'expérimentations effectuées sur la température cutanée se rapportent à des ambiances particulières pour lesquelles l'organisme est en situation d'adaptation (approche vers une situation de confort) ou de défense (protection contre une situation mauvaise), mais pas de confort. Pour de telles ambiances, la température radiante a toujours été prise voisine de la température de l'air et l'habillement réduit $(<0,6 \mathrm{clo})$.

L'usage des expressions de $T_{\text {sk }}$ proposées par les divers auteurs doit donc être fait avec précautions en respectant les limites d'application et les conditions d'expérimentation. Les diagrammes de confort ci-après sont en conséquence volontairement bornés à la zone dite de confort neutre ou optimal; néanmoins, la zone $T_{\text {air }}=T_{\text {radiante }}$ peut être complétée par celle $T_{\text {operative }}=$ constante en raison d'une certaine impossibilité de l'organisme à distinguer entre échanges convectifs et radiatifs : en effet, d'une part les terminaisons sensitives ne se trouvent pas en surface de la peau, mais au-dessous, d'autre part le port du vêtement amène les échanges extérieurs à s'effectuer au niveau de la peau sous forme conductive. On retiendra cependant que l'oil est, en tant que corps noir à $37^{\circ}$, l'organe de perception des ambiances radiatives, tandis que le nez est l'organe d'appréciation des ambiances convectives.

Les zones que nous appellerons de confort instable, au-delà des nœuds sur clo et $V_{\text {air }}$ [9], existent cependant, mais nécessitent des expérimentations pour être décrites. Les zones d'inconfort (trop froides ou trop chaudes) peuvent être analysées sur le plan " réactions de l'organisme, et actions sur les paramètres pour une approche vers des situations moins inconfortables ", mais ceci n'entre pas dans le cadre de cet article consacré aux ambiances de confort.

3.1.2 La température cutanée moyenne. - Les différentes parties du corps humain ne sont pas à la même température $[21,22]$. La vasomotricité module les échanges et conduit à des différences notables sur la température cutanée. Ceci amène d'une part à prendre en compte les parties non habillées (tête et mains, soit $6 \%$ de la surface du corps) avec leur température propre pour les échanges avec l'extérieur, d'autre part à définir une température cutanée par une moyenne pondérée des différentes températures du corps [21]. Dans la zone dite de confort ( 10 à $30^{\circ}$ ) cette température se situe entre la température du noyau central du corps et celle de l'extérieur pour que soit assurée la dépense énergétique correspondant au métabolisme. C'est pourquoi elle varie en sens contraire du niveau d'activité. Cependant, lorsque celui-ci devient trop grand, ou que les conditions extérieures sont trop éloignées des conditions de confort, la peau ne parvient plus à constituer un régulateur des échanges. Elle suit alors la température du noyau ( $\left.T_{\text {ambiance }}>34\right)$ ou est dominée par l'influence de l'ambiance si celle-ci est trop froide (contraction des vaisseaux qui diminue le flux sanguin, celui-ci étant le fluide caloporteur). Dans ces cas-là, on ne peut plus parler de confort. 
La température d'ambiance, ainsi que l'hygrométrie de l'air influent sur $T_{\text {sk }}$ pour que soient ajustés convenablement les échanges par convection, rayonnement et sudation. Cependant, lorsque la mouillure de la peau devient trop grande, on quitte une situation de confort : la sudation ne régule plus les échanges et la dépendance de $T_{\mathrm{sk}}$ vis-à-vis de la pression de vapeur d'eau de l'ambiance s'annule.

La température de la peau est un paramètre fondamental pour l'établissement des diagrammes de confort. Les zones de confort correspondent d'ailleurs aux cas où cette température peut s'établir d'elle-même sur une valeur qui assure un bilan énergétique nul pour l'organisme. Il n'y a alors aucune domination des températures du noyau central ou de l'ambiance, et les processus d'échanges ont leur efficacité maximale.

Le cas des climats chauds est différent de celui traité ici puisqu'il faut tenir compte très particulièrement des températures de peau et du noyau central, toutes deux liées l'une à l'autre. L'évaporation en dépend et la mouillure devient le critère de confort.

3.1.3 Les expressions de $T_{\text {sk }}$ proposées. - L'adoption d'une expression satisfaisante pour $T_{\text {sk }}$ pose des problèmes en raison des difficultés qu'il $\mathrm{y}$ a pour confronter les formulations proposées par les différents auteurs :

- Les travaux de Fanger $[2,19]$ sur 183 sujets ont abouti à une expression en fonction du seul paramètre activité :

$$
T_{\text {sk }}=(35,7-0,037) 58,15 A C T .
$$

L'avantage de cette expression est d'avoir été établie pour des situations de confort; pour obtenir de telles situations, l'auteur a fait varier les autres paramètres en fonction de l'activité, ce qui lie $T_{\text {sk }}$ à ces paramètres implicitement. Cependant, des conditions très diverses peuvent être obtenues pour une même activité, tout en assurant une situation de confort. La relation de Fanger ne permet pas de les distinguer, bien qu'elles puissent être associées à des valeurs différentes de $T_{\text {sk }}$. C'est pourquoi cette relation doit être considérée pour la seule zone $T_{\text {air }}$ voisin de $T_{\text {radiante }}$ pour laquelle elle a été établie. La forme du nuage de points sur lequel cette expression a été établie [19] est d'ailleurs très ovale, ce qui montre l'imprécision de cette relation.

- Un certain nombre d'expressions [21,23] ont été établies par des expérimentations sur 3 ou 4 sujets, ou pour des conditions hors du confort : elles ne doivent donc être considérées que comme des confirmations ou des compléments d'information. L'influence du vêtement a été peu étudiée [24] et n'apparaît pas dans les formulations, ce qui est regrettable : le " clo " généralement adopté est faible, inférieur à 0,5 .

- Les travaux de Meyer [21] englobent des zones de confort et d'inconfort. Etablis à partir de 29 sujets expérimentaux (ayant conduit à l'analyse de 2987 valeurs de la température cutanée moyenne), ils aboutissent à 7 formulations, 3 hors de la zone de confort, 4 correspondant aux conditions d'activité et d'acclimatation :

\begin{tabular}{l|c}
\multicolumn{1}{c|}{$T_{\text {sk }}$} & Sujets non acclimatés \\
\hline Repos & $34,9-0,251\left(30-T_{\mathrm{A}}\right)$ \\
Travail & $33,0-0,240\left(30-T_{\mathrm{A}}\right)+0,076 \mathrm{~Pa}$
\end{tabular}

où $T_{\mathrm{A}}$ est une certaine température d'ambiance correspondant le plus souvent à $T_{\text {air }}=T_{\text {rad }}=T_{\mathrm{A}}$.

Il est intéressant que l'acclimatement ait été considéré, car il induit des modifications des phénomènes physiologiques qui permettent à l'homme de vivre en homéostasie thermique : diminution des températures centrale et périphérique, de la fréquence cardiaque, performances sudorales améliorées. Le cas des sujets acclimatés sera seul considéré ici puisqu'on cherchera des situations de confort reproductibles.

Meyer propose aussi une relation simplifiée, indépendante de $P a$, paramètre dont l'influence est difficile à exprimer :

$$
T_{\text {sk }}=28,65+0,18 T_{\mathrm{A}} .
$$

Une comparaison entre différentes relations est donnée par le tableau III et la figure 3.

Les résultats donnés par la relation de GonzalezNishi-Gagge [25] pour les faibles activités, et ceux donnés par la relation (38) de Meyer paraissent les meilleurs pour être en accord à la fois avec la relation de Fanger et la réalité.

Ils permettent de proposer une expression pour la relation de Fanger faisant intervenir, non seulement l'activité, mais aussi la température d'ambiance et l'habillement :

$$
\begin{aligned}
T_{\text {sk }}=29,55+0,196 T_{\mathrm{A}}- & 1,064 \mathrm{ACT} \times \\
& \times\left(1-0,295 \mathcal{R}_{\mathrm{clo}}\right) .
\end{aligned}
$$

Une telle relation ne correspond qu'à une construction mathématique : dans cette relation, tout comme dans celle de Fanger, les paramètres doivent être en accord avec $V_{\text {air }}$ et $\phi$ pour correspondre à une situation de confort. Ces deux relations restent approchées pour deux raisons :

- Il existe plusieurs valeurs de $T_{\mathrm{A}}$ en accord avec une même combinaison des autres paramètres et satisfaisant le confort. 
Tableau III. - Température d'ambiance souhaitée pour une situation de confort (accord avec la relation de Fanger).

\begin{tabular}{c|ccccccc}
$T_{\text {A }}$ & clo $=0$ & 1 & $0,6 ?$ & $0,6 ?$ & $0,5 ?$ & $0,5 ?$ & $0,5 ?$ \\
\hline$A C T=0,8$ & 27,9 & 26,7 & 27,1 & 27,1 & 32,7 & 33,8 & \\
2 & 20,2 & 17,0 & 17,5 & 17,2 & 23,5 & 21,8 & 21,1 \\
3 & 14,7 & 9,9 & 9,8 & 4,6 & 15,8 & 11,8 & 10,7 \\
& Gonzalez & & & & Rublack & Saltin & Candas \\
& Nishi & & $(35)$ & $(34)$ & Shultz & Gagge &
\end{tabular}

(36) (36)

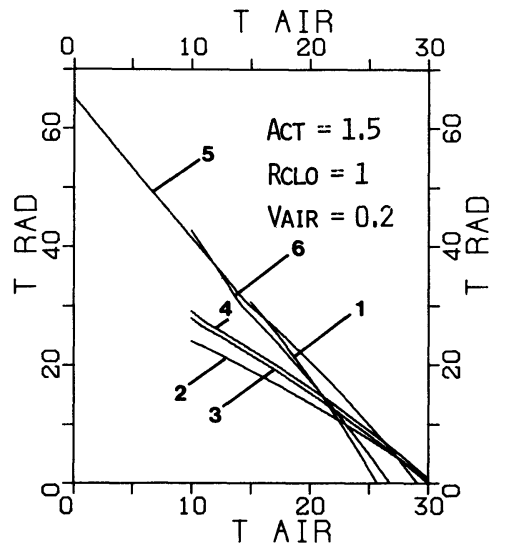

Fig. 3. - Courbes de confort établies à partir de différentes formulations de la température moyenne cutanée.

(1) Formule de Gonzalez, Nishi et Gagge (1975) :

$$
T_{\text {sk }}=29,55+0,196 T_{\text {op }}-0,0183 M_{\mathrm{T}} / A_{\mathrm{DU}} .
$$

(2) Formule de Rubback et Shultz (1974) :

$$
T_{\text {sk }}=24,8+0,28 T_{\mathrm{A}} .
$$

(3) Formule de Saltin et Gagge (1971) :

$$
T_{\text {sk }}=26,7+0,215 T_{\mathrm{A}} .
$$

(4) Formule de Candas (1974)

$$
T_{\text {sk }}=27,0+0,208 T_{\text {A }} .
$$

(5) Formule de Fanger (1967) :

$$
T_{\text {sk }}=35,7-0,037 M_{\mathrm{T}} / A_{\mathrm{DU}} .
$$

(6) Formule de Meyer (1981) :

$$
T_{\text {sk }}=32,5-0,166\left(30-T_{\text {op }}\right)+0,08 \frac{1013}{760} P a .
$$

[Comfort lines established with different mean skin temperature relations.]

- Une double expression de $T_{\text {sk }}$ lie entre eux les paramètres : mais la liaison simple donnée cache cependant l'ensemble des équations des échanges énergétiques qui expriment la situation de confort et dont la solution n'a rien de simple : c'est pourquoi, tant l'une que l'autre, ces expressions ne doivent être considérées que comme des informations uniquement valables dans la zone de confort voisine de $T_{\text {air }}=T_{\text {rad }}$. Pour les ambiances de confort radiativement déséquilibrées les expérimentations restent à faire car il n'y en a pas eu, et une extrapolation pour $T_{\text {op }}=$ Cte loin de la zone $T_{\mathrm{A}}=T_{\text {rad }}$ reste sujette à contestation. Pour les zones qui ne sont pas de confort, rappelons qu'aucune extrapolation de ces relations ne peut être faite, car le comportement de la peau en situation de défense est tout autre.

\section{2 LE VÊTEMENT.}

3.2.1 La température du vêtement. - Le vêtement a une importance capitale dans la notion de confort et son action est souvent multiple. Il peut nous protéger $\mathrm{du}$ froid par son pouvoir isolant, de la pluie par ses propriétés imperméables, du rayonnement trop fort du soleil en pays chaud...

La production de chaleur interne du corps humain peut varier de quelque $40 \mathrm{~W} / \mathrm{m}^{2}$ pour un sujet allongé au repos à des valeurs de l'ordre de $600 \mathrm{~W} / \mathrm{m}^{2}$ ou plus pour des activités physiques intenses (travaux de force, sport de compétition...). Cette chaleur doit être dissipée par les différents modes précédemment étudiés et le vêtement adéquat à chaque situation d'activité et d'ambiance thermique doit permettre d'équilibrer le bilan thermique dans de bonnes conditions.

Il doit, par ailleurs, assurer d'autres fonctions (être agréable à porter, être perméable à la transpiration...). Le vêtement sera ici considéré comme paramètre intervenant dans les échanges énergétiques en climat tempéré ou froid, les fonctions émissives ou soufflet étant spécifiques des climats chauds hors de la zone de confort et non considérées ici.

Il apparaît que la grandeur caractéristique qui intervient dans le calcul des échanges radiatifs et convectifs entre l'homme habillé et son environnement est la température moyenne de surface du vêtement $T_{\text {clo }}$.

La résistance thermique des vêtements est usuellement repérée dans une échelle en " clo ".

$$
1 \text { clo }=0,155 \mathrm{~m}^{2} .{ }^{\circ} \mathrm{C} / \mathrm{W} \text {. }
$$

Le tableau IV donne l'isolation en clo de quelques tenues standards. 
Tableau IV.

\begin{tabular}{|lc|}
\hline \multicolumn{1}{c}{ Description de l'habillement } & Isolation (clo) \\
$\mathrm{Nu}$ & 0 \\
Sous-vêtements & 0,1 \\
Tenue tropicale type & $0,3-0,4$ \\
(short, tee-shirt à manches courtes, socquettes et sandales) & 0,5 \\
$\begin{array}{l}\text { Tenue d'été légère } \\
\text { (sous-vêtements courts, pantalons légers, tee-shirt à manches courtes) }\end{array}$ & 0,6 \\
$\begin{array}{l}\text { Tenue de travail légère } \\
\text { (sous-vêtements courts, chaussettes coton, pantalon de travail, maillot coton sur le panta- }\end{array}$ & \\
$\quad$ lon) & 0,8 \\
Costume d'été & 1,0 \\
Costume de travail + pardessus & 1,5 \\
Tenue de sport d'extérieur & $0,8-1,0$ \\
Costume d'hiver avec sous-vêtements chauds + imperméable & 1,5 \\
\hline
\end{tabular}

Si $\mathcal{R}$ est la résistance thermique du vêtement et $\mathcal{R}_{\text {clo }}$ son isolation exprimée en clo, on a évidemment :

$$
\mathcal{R}=0,155 \mathcal{R}_{\text {clo }} .
$$

Soit $K\left(\mathrm{~W} / \mathrm{m}^{2}\right)$ le flux conductif à travers le vêtement. On a le schéma suivant :

$$
\begin{aligned}
& A C T
\end{aligned}
$$

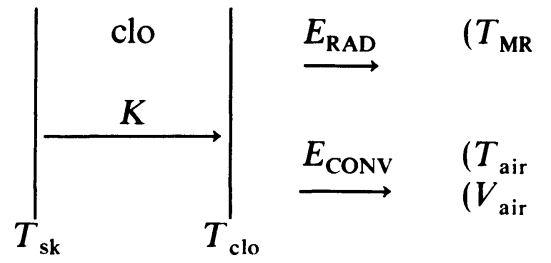

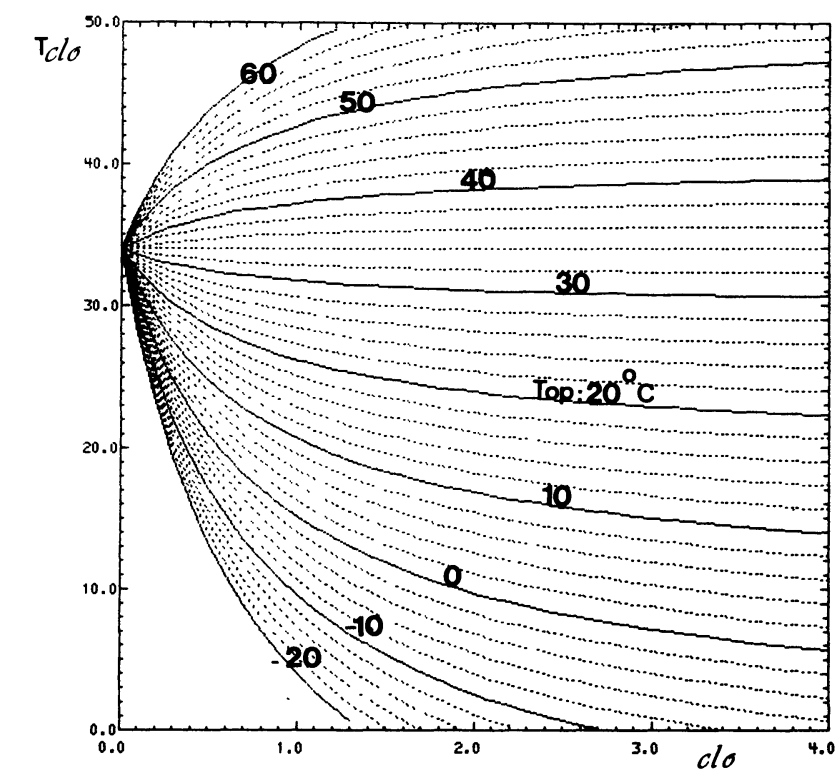

soit en explicitant chaque terme :

$$
\frac{T_{\text {clo }}-T_{\text {sk }}}{0,155 \mathcal{R}_{\text {clo }}}=h_{\mathrm{R}}\left(T_{\mathrm{MR}}-T_{\text {clo }}\right)+h_{\mathrm{C}}\left(T_{\text {air }}-T_{\text {clo }}\right) .
$$

Ce qui conduit directement à l'expression de la température de surface du vêtement :

$$
T_{\text {clo }}=\frac{\left(h_{\mathrm{R}} T_{\mathrm{MR}}+h_{\mathrm{C}} T_{\mathrm{air}}\right) 0,155 \mathcal{R}_{\mathrm{clo}}+T_{\mathrm{sk}}}{1+\left(h_{\mathrm{R}}+h_{\mathrm{C}}\right) 0,155 \Re_{\mathrm{clo}}} .
$$

On remarque que la fonction $T_{\text {clo }}=f\left(\mathcal{R}_{\text {clo }}\right)$ (Fig. 4) est une hyperbole (toutes choses restant égales par ailleurs).

$$
\lim _{\mathcal{R}_{\mathrm{clo}} \rightarrow \infty} T_{\mathrm{clo}}=\frac{h_{\mathrm{R}} T_{\mathrm{MR}}+h_{\mathrm{C}} T_{\mathrm{air}}}{h_{\mathrm{R}}+h_{\mathrm{C}}}=T_{\mathrm{op}} .
$$

L'asymptote horizontale n'est autre que la température opérative $T_{\mathrm{op}}$ définie par Gagge en 1940 [26].
Fig. 4. - Température moyenne de surface des vêtements en fonction de l'isolation du vêtement, pour différentes valeurs de la température opérative.

[Mean temperature of outer surface of clothed body $\left(T_{\text {clo }}\right)$ as a function of thermal resistance (clo) from skin to outer surface of the clothed body, at different operative temperatures $\left(T_{\text {op }}\right)$.]

Cette température correspond à la température limite d'un vêtement de résistance thermique infinie, et donc à un échange global sec nul avec l'extérieur, ce qui ne saurait être un état de confort thermique. Par la suite, nous garderons l'être mathématique $T_{\text {op }}$ qui nous permettra de faciliter le repérage d'une ambiance.

3.2.2 Influence de $T_{\text {clo }}$ sur les échanges énergétiques. La température moyenne cutanée est de l'ordre de 
$34^{\circ} \mathrm{C}$. Nous avons montré en 3.1 qu'elle varie avec les autres paramètres mais pour une compréhension de l'influence de $T_{\text {clo }}$, cette valeur nous conviendra.

Analysons les 8 cas de figures possibles, les cas 1 à 4 correspondent à une température opérative inférieure à la température moyenne cutanée. Ce sont les plus courants en climat tempéré excepté le cas 4 qui correspond à une température d'air supérieure à $34^{\circ} \mathrm{C}$ avec un faible rayonnement. $\mathrm{Ce}$ cas théorique peut correspondre à une climatisation radiative froide en pays méditerranéen.

Les cas 5 à 8 peuvent se produire en climat tropical et sont indiqués ici comme cas théoriques. L'analyse du climat tropical fera l'objet d'une étude ultérieure.

Cas 1: $T_{\text {air }}<T_{\text {MR }}<T_{\text {sk }}$ (Fig. 5).

Jusqu'à $\mathcal{R}_{\text {clo }_{1}}$, les échanges radiatifs et convectifs correspondent à une perte pour l'organisme et diminuent en valeur absolue quand $\mathcal{R}_{\text {clo }}$ augmente. Il existe une valeur de l'isolation $\mathcal{R}_{\text {clo }}=\mathcal{R}_{\text {clo }_{1}}$, pour laquelle les échanges radiatifs sont nuls. Au-delà de cette valeur, les échanges radiatifs sont un gain pour l'organisme. A l'infini, $E_{\mathrm{RAD}}$ et $E_{\mathrm{CONV}}$ se compensent et leur action simultanée s'annule.

Pour déterminer cette valeur «charnière» de l'isolation, il suffit de poser $T_{\text {clo }}=T_{\mathrm{MR}}$ dans (41). Il vient :

$$
R_{\mathrm{clo}_{1}}=\frac{T_{\text {sk }}-T_{\mathrm{MR}}}{T_{\mathrm{MR}}-T_{\mathrm{air}}} \times \frac{1}{0,155 h_{\mathrm{C}}} .
$$

Cas 2: $T_{\text {air }}<T_{\text {sk }}<T_{\text {MR }}$ (Fig. 6).

Dans ce cas, quelle que soit l'isolation du vêtement porté, les échanges radiatifs sont un gain alors que les échanges convectifs sont une perte pour l'organisme. Ce cas se rencontre fréquemment en extérieur, sous nos latitudes, par des journées ensoleillées à mi-saison.

Cas 3: $T_{\text {MR }}<T_{\text {air }}<T_{\text {sk }}$ (Fig. 7).

Détermination de $\mathcal{R}_{\text {clo }_{3}}$ (habillement correspondant à l'annulation des échanges convectifs, soit $T_{\text {air }}=$ $\left.T_{\text {clo }}\right)$.

De la même manière que pour le cas 1 , on trouve :

$$
\mathcal{R}_{\mathrm{clo}_{3}}=\frac{T_{\mathrm{sk}}-T_{\mathrm{air}}}{T_{\mathrm{air}}-T_{\mathrm{MR}}} \times \frac{1}{0,155 h_{\mathrm{C}}} .
$$

Cas 4 : $T_{\text {MR }}<T_{\text {sk }}<T_{\text {air }}$ (Fig. 8).

Cas 5: $T_{\text {sk }}<T_{\text {air }}<T_{\text {MR }}$ (Fig. 9).

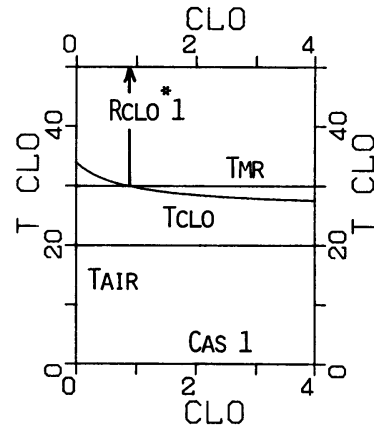

Fig. 5.

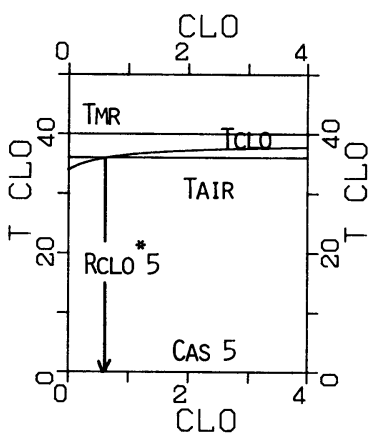

Fig. 9.

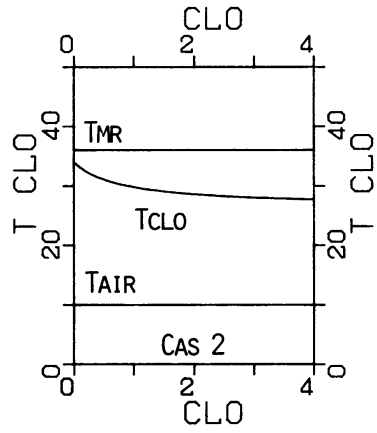

Fig. 6.

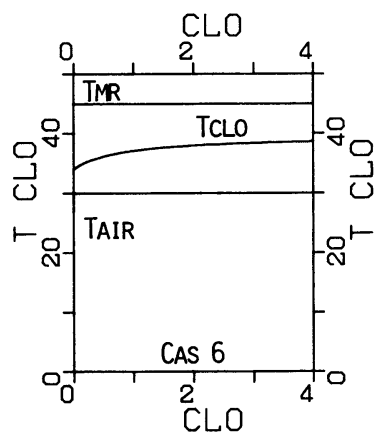

Fig. 10 .

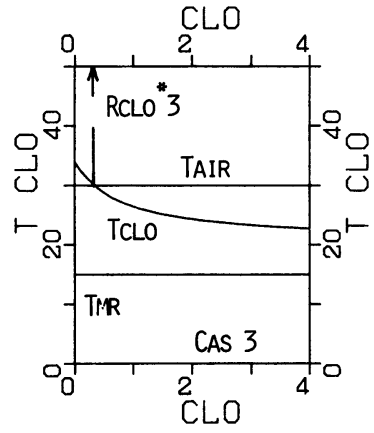

Fig. 7.

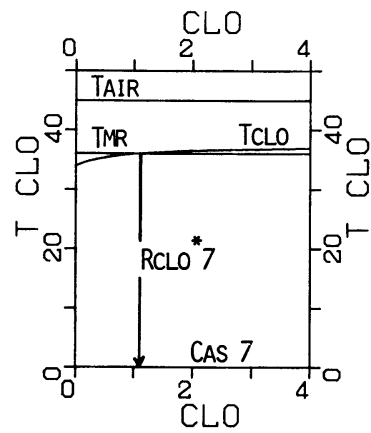

Fig. 11 .

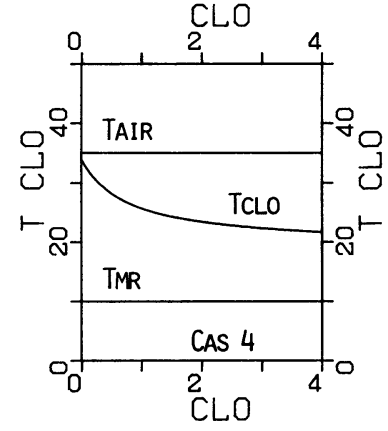

Fig. 8.

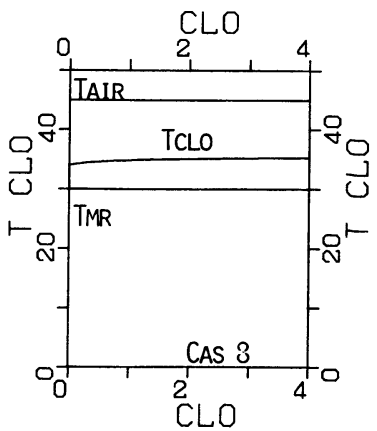

Fig. 12.

Figs. 5 à 12. - Cas 1 à 8 (voir 3.2.2) : différents cas d'ambiances thermiques montrant, en fonction de l'isolation thermique du vêtement, les positions relatives de la température moyenne radiante, de la température d'air et de la température de surface extérieure du vêtement.

[Case 1 to 8 (see 3.2.2) : different sets of thermal ambiances showing the relative positions of the mean radiant temperature, of the air temperature, and of the outer surface of clothing.] 
L'habillement d'isolation $\mathcal{R}_{\text {clos }_{5}}$ correspond à des échanges convectifs nuls soit $T_{\text {clo }}=T_{\text {air }}$.

$$
\mathcal{R}_{\mathrm{clo}_{5}}=\frac{T_{\mathrm{air}}-T_{\mathrm{sk}}}{T_{\mathrm{MR}}-T_{\mathrm{air}}} \cdot \frac{1}{0,155 h_{\mathrm{C}}} .
$$

Cas 6 : $T_{\text {air }}<\mathrm{T}_{\text {sk }}<\mathrm{T}_{\mathrm{MR}}($ Fig. 10).

Cas 7: $T_{\text {sk }}<T_{\text {MR }}<T_{\text {air }}$ (Fig. 11).

Détermination de $\mathcal{R}_{\mathrm{clo}_{7}}$ : les échanges radiatifs sont nuls, ce qui impose $T_{\text {clo }}-T_{\text {air }}$, d'où l'on déduit immédiatement :

$$
\mathcal{R}_{\mathrm{clo}_{7}}=\frac{T_{\mathrm{MR}}-T_{\mathrm{sk}}}{T_{\mathrm{air}}-T_{\mathrm{MR}}} \cdot \frac{1}{0,155 h_{\mathrm{C}}} .
$$

Cas 8 $: T_{\mathrm{MR}}<T_{\text {sk }}<T_{\text {air }}$ (Fig. 12).

Pour chaque cas, il a été traité un exemple :

$\begin{array}{lcccccccc}\text { Cas } & 1 & 2 & 3 & 4 & 5 & 6 & 7 & 8 \\ - & - & - & - & - & - & - & - & - \\ T_{\text {air }} & 20 & 10 & 30 & 35 & 36 & 30 & 45 & 45 \\ T_{\text {MR }} & 30 & 36 & 15 & 10 & 40 & 45 & 36 & 30 \\ T_{\text {op }} & 26,3 & 26,5 & 20,5 & 19,2 & 38,5 & 39,5 & 37,5 & 35,5\end{array}$

Les courbes 13 à 20 donnent les échanges radiatifs $R$ en watts, les échanges convectifs $C(\mathrm{~W})$ - et leur somme $T=C+R$ en fonction de la résistance thermique des vêtements en clo, pour une activité de 1,5 Met, et une vitesse d'air de $0,2 \mathrm{~m} / \mathrm{s}$.

\section{Résultats du modèle. Courbes de confort.}

4.1 COURBES DE CONFORT. - Les formules choisies et discutées plus haut ont permis d'élaborer un programme informatique décomposé en deux parties : le bloc calcul, qui détermine le bilan énergétique (soit un stockage de l'organisme) et le bloc qui optimise la valeur d'une des six variables d'entrées indépendantes (température de l'air, température moyenne radiante, hygrométrie, vitesse relative de l'air, activité $\mathrm{du}$ sujet ainsi que la résistance thermique de son vêtement) pour annuler le bilan.

Les résultats sont présentés ici sous la forme de douze courbes (Fig. 21 à Fig. 32) pour une hygrométrie de $50 \%$. Le système d'axes $\left(T_{\mathrm{A}}, T_{\mathrm{MR}}\right)$ a été choisi pour faire ressortir l'effet des températures. Un point du diagramme caractérise un état d'agrément, ou plutôt une situation d'ambiance de confort. Les deux figures suivantes sont des représentations tridimensionnelles qui permettent d'observer l'influence de la vitesse de l'air (Fig. 33) et de l'habillement (Fig. 34).

Le tableau V est le résultat d'une version " conversationnelle " du programme et donne pour un cas particulier d'ambiance thermique et des caractéristiques personnelles du sujet, la valeur des différents termes du bilan.

\subsection{INFLUENCE DES DIFFÉRENTS PARAMÈTRES.}

4.2.1 La vitesse de l'air. - Les courbes de confort (Fig. 21 à Fig. 32) laissent apparaître un "nœeud »

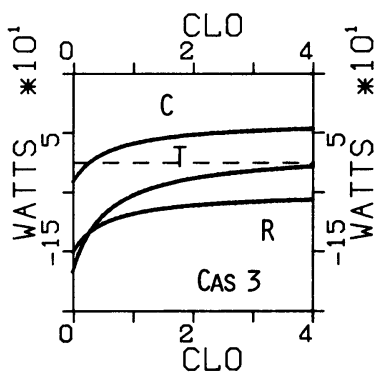

Fig. 15.

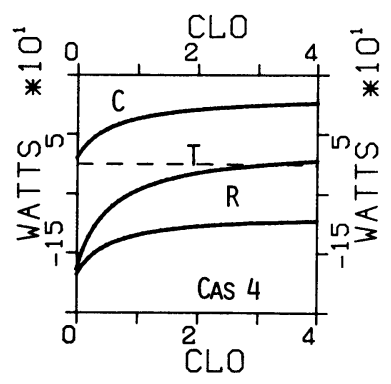

Fig. 16.
Fig. 13.

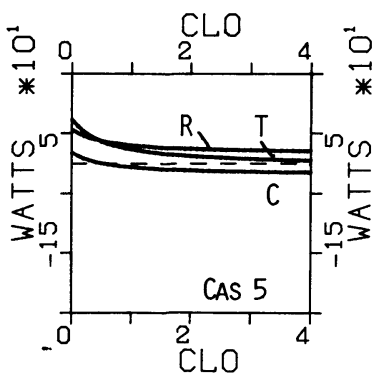

Fig. 17.

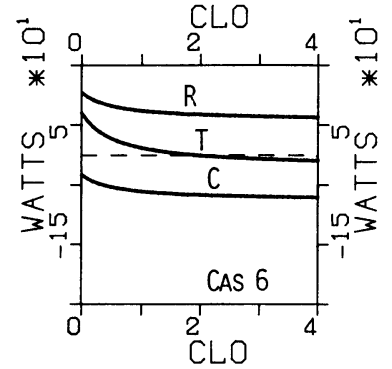

Fig. 18 .

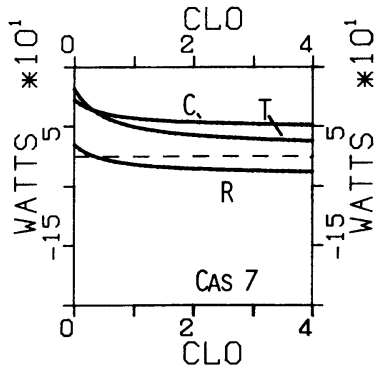

Fig. 19.

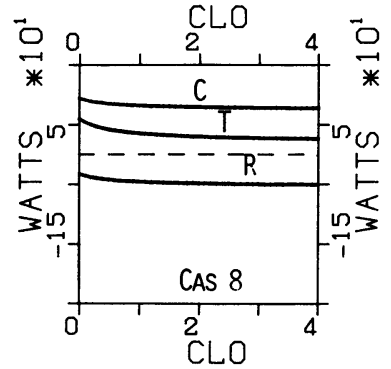

Fig. 20.

Figs. 13 à 20. - Echange radiatif $(R)$, convectif $(C)$ et global $\sec (T=R+C)$ pour les différents cas possibles (5 à 12).

[Radiative $(R)$, convective $(C)$ and global dry $(T=R+C)$ heat exchanges for the different considered cases (see Figs. 5 to 12$)]$ 


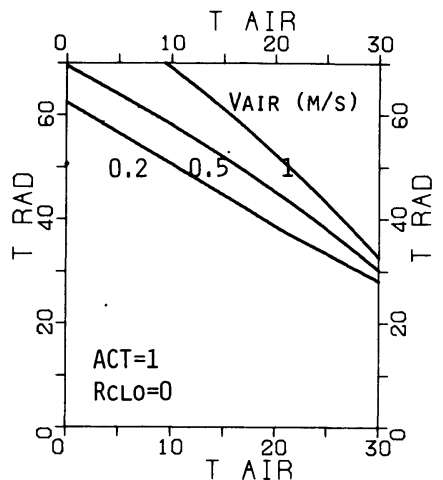

Fig. 21.

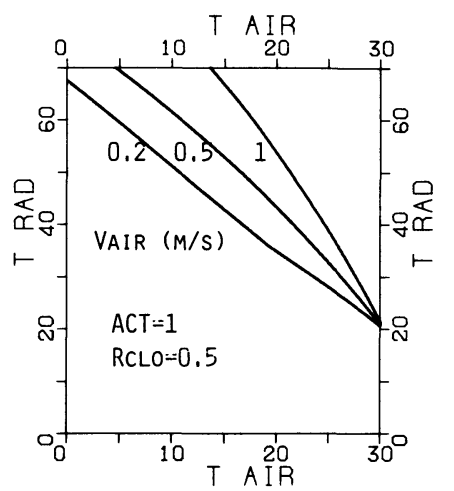

Fig. 22.

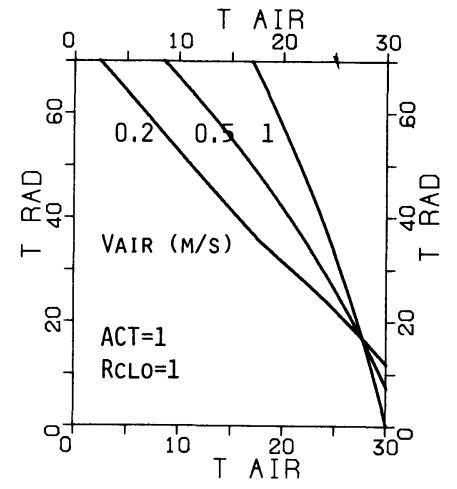

Fig. 23.

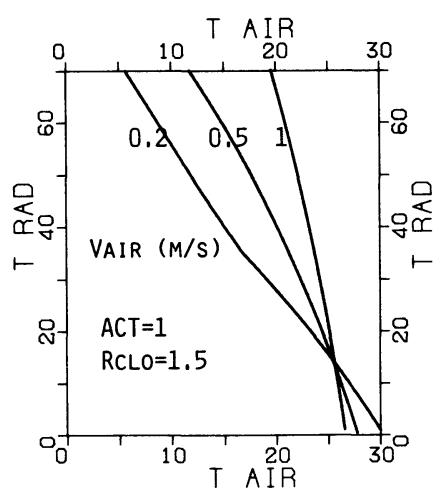

Fig. 24.

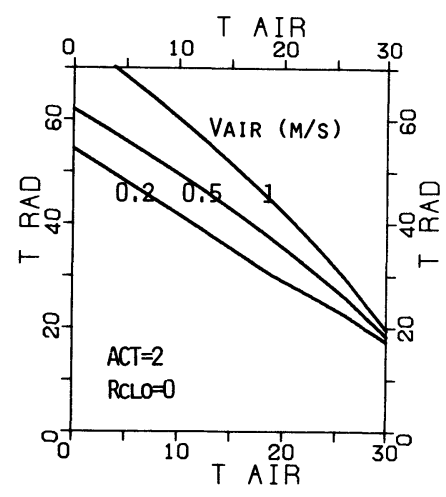

Fig. 25.

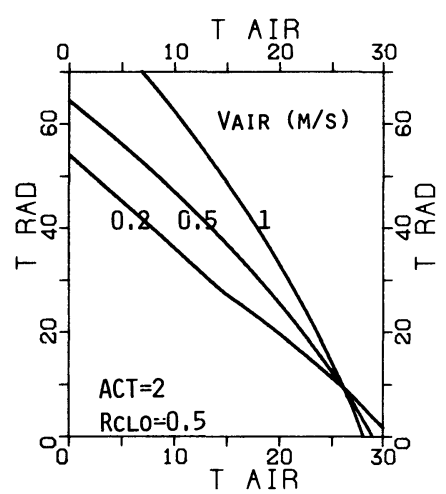

Fig. 26.

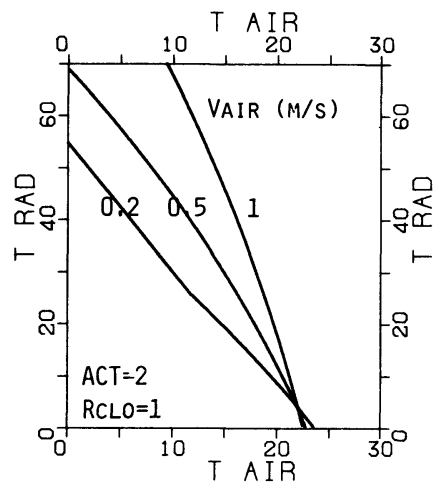

Fig. 27.

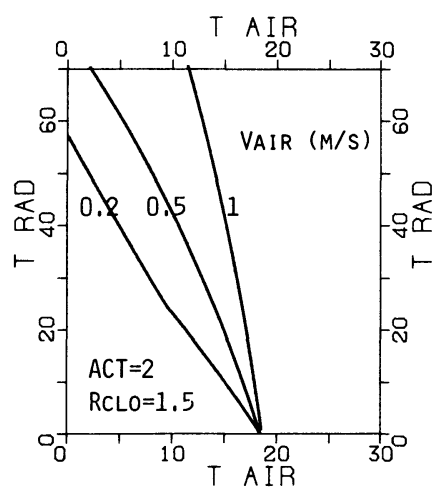

Fig. 28.

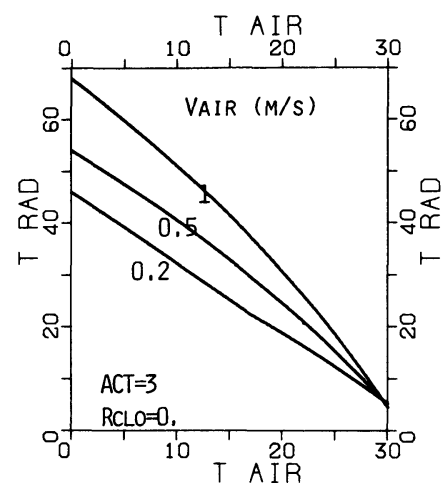

Fig. 29.

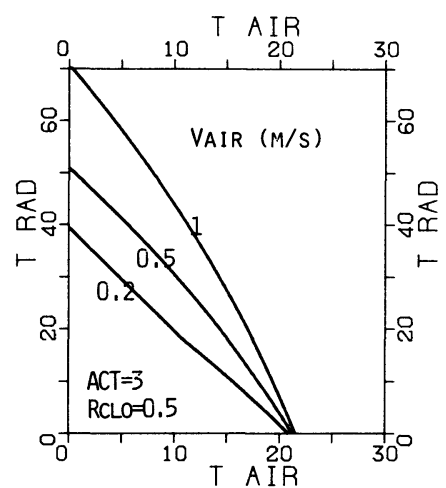

Fig. 30 .

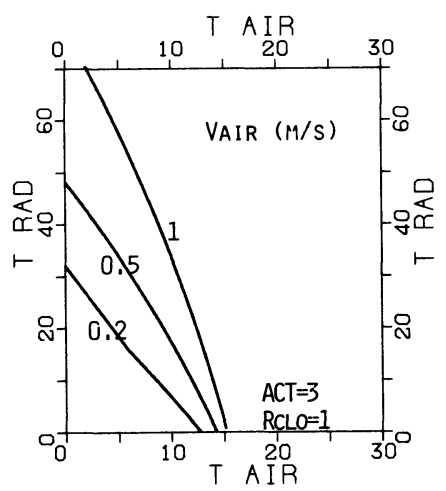

Fig. 31 .

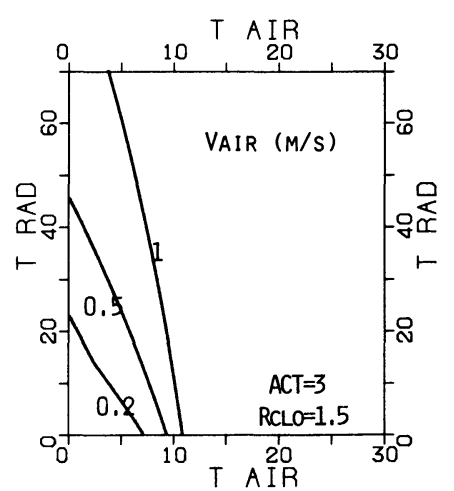

Fig. 32.

Figs. 21 à 32. - Courbes de confort pour une hygrométrie de l'air de $50 \%$ (température moyenne radiante en fonction de la température d'air) paramétrées en fonction de la vitesse relative de l'air pour des individus ayant une activité donnée $A C T$ et une isolation vestimentaire donnée $\mathcal{R}_{\text {clo }}$.

[Comfort diagrams (air temperature versus mean radiant temperature with relative air velocity as parameter) for several clothing $\left(\mathcal{R}_{\text {clo }}\right)$ and activities $(A C T)$ given values.] 
Tableau V. - Bilan (exemple).

Cas d'un sujet debout mesurant

Son poids est de

Son activité correspond à

Son habillement correspond à

Température d'air

Température moyenne radiante

Humidité relative

Vitesse relative de l'air

Début des calculs

Poids en $\mathrm{g}$ d'eau par kg d'air sec

Température humide

Température de rosée

Température moyenne cutanée

Température de l'air expiré

Température des vêtements

Métabolisme énergétique total

Echanges de chaleur radiatifs

Echanges de chaleur convectifs

Echanges respiratoires par évaporation

Echanges respiratoires par convection

Echanges respiratoires par diffusion

Evaporation de la sueur

Stockage dans l'organisme
$1,70 \mathrm{~m}$

$70,0 \mathrm{~kg}$

1,6 Met

1,0 clo

$19,20^{\circ} \mathrm{C}$

$19,20^{\circ} \mathrm{C}$

$50,00 \%$

$0,20 \mathrm{~m} / \mathrm{s}$

$6,97 \mathrm{~g} / \mathrm{kg}$

$13,09^{\circ} \mathrm{C}$

$8,39^{\circ} \mathrm{C}$

$32,15^{\circ} \mathrm{C}$

$34,09^{\circ} \mathrm{C}$

$24,11^{\circ} \mathrm{C}$

$174,09 \mathrm{~W}$

$-48,59 \mathrm{~W}$

$-52,17 \mathrm{~W}$

$-14,94 \mathrm{~W}$

$-4,15 \mathrm{~W}$

$-20,59 \mathrm{~W}$

$-33,60 \mathrm{~W}$

$0,05 \mathrm{~W}$

d'indifférence pour les fortes températures de l'air. En ce point, la température des vêtements $T_{\text {clo }}$ atteint la température de l'air et les échanges sont uniquement radiatifs (ceci n'est bien entendu pas réalisé localement au niveau des parties découvertes du corps). Cette situation correspond au point $\mathcal{R}_{\mathrm{clo}_{3}}$ de la figure 7 . Les ensembles de nœuds pour clo et $\phi$ variables constituent une barrière remarquable. Sur la figure 33, on a indiqué en trait épais le lieu des nœuds d'indifférence pour des valeurs données de l'activité, de l'habillement et de l'hygrométrie.

Cette situation s'obtient avec des faibles températures moyennes radiantes associées à des fortes températures d'air : c'est le cas des climatisations radiatives froides. Techniquement, on peut noter que l'on doit associer ces combinaisons à des hygrométries faibles afin que la température de rosée ne dépasse pas la température des parois. D'un point de vue physiologique, on remarque un phénomène qui peut entraver l'efficacité d'un tel système. En effet, il apparaît que si l'on se place sur le diagramme à une forte température d'air, aux alentours de ce " noud » (point où le confort est indifférent à la vitesse de l'air) un accroissement du métabolisme va provoquer un déplacement du " nœud" vers les plus faibles températures d'air, provoquant aussitôt une sensation d'inconfort : l'augmentation du métabolisme ne sera pas compensée par les pertes ; les échanges convectifs seront un apport. Autrement dit, tout travail dans ces zones de températures provoque un désagrément qui amènera le

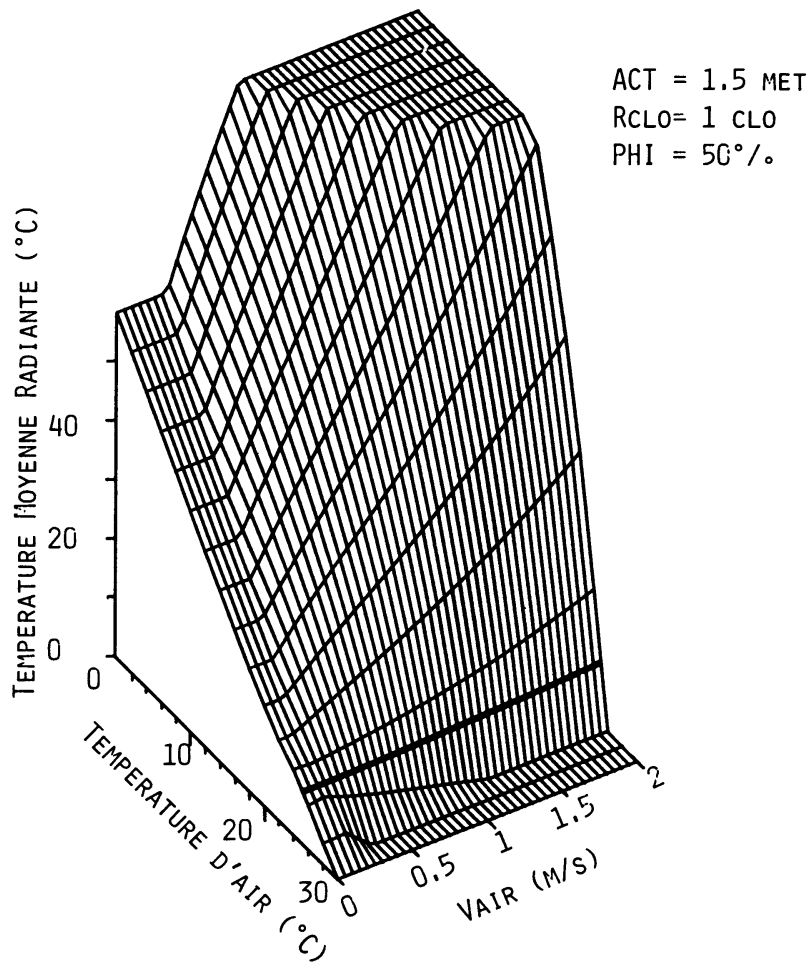

Fig. 33. - Diagramme de confort tridimensionnel (température d'air, température moyenne radiante, vitesse relative de l'air) pour une activité de 1,5 Met, une isolation thermique due au vêtement de 1 clo et une hygrométrie de l'air de $50 \%$. En trait épais : l'indifférence à la vitesse de l'air.

[Tridimensional comfort diagram (air temperature, mean radiant temperature and relative air velocity for a $1.5 \mathrm{Met}$ activity, 1 clo insulation and $50 \%$ air hygrometry. In thick line, the non influence of the air velocity.]

sujet à modifier son comportement et réduire son activité (sieste en pays chaud).

Au niveau du palier que l'on peut observer sur la figure 33, pour les faibles vitesses d'air (inférieures à $0,2 \mathrm{~m} / \mathrm{s}$ ), l'influence des écarts de températures (individu-environnement) est prépondérante sur la vitesse de l'air. La nappe est volontairement coupée pour les fortes températures moyennes radiantes, la validité du modèle étant contestable dans ces zones où les expérimentations sont inexistantes. Dans les cas courants, il faudra éviter, dans la mesure du possible, des vitesses d'écoulement supérieures à $0,4 \mathrm{~m} / \mathrm{s}$. Audelà de cette valeur, les " courants d'air " sont perçus comme désagréables et peuvent occasionner des troubles pathologiques.

Cependant, cette vitesse peut être supérieure dans les cas d'un air soufflé (envoi d'un air à une température différente de celle de l'ambiance) ou d'un air chaud, inférieur toutefois à $35^{\circ}$ environ [27]. Dans ce dernier cas ce n'est plus l'échange convectif qui est à considérer, mais celui évaporatif qui doit être obtenu à son maximum d'efficacité ; $1 \mathrm{~m} / \mathrm{s}$ est alors une valeur possible à adopter. 
4.2.2 L'habillement. - La caractéristique du vêtement qui apparaît dans le modèle est la résistance thermique $\mathcal{R}_{\text {clo }}$. Cela n'est pas suffisant mais ce choix délibéré correspond à un souci de simplicité et de reproductibilité des situations. L'adjonction d'un paramètre tel que la perméabilité du vêtement à la vapeur d'eau aurait multiplié les cas possibles et alourdi l'analyse. Toutefois, la version " conversation " du programme permet cette étude cas par cas. Une mise en garde importante est à faire à propos de $\mathcal{R}_{\text {clo. }}$. Les courbes à clo constant ne correspondent pas nécessairement à un vêtement donné. En effet, pour des fortes teneurs en eau dans l'atmosphère et des températures d'air basses, le matériau textile est chargé en eau et perd ses qualités d'isolation. D'autre part, en fonction de l'ampleur du vêtement, un effet dit " soufflet » vient modifier les processus de transfert thermique et occasionner des variations sensibles sur $\mathcal{R}_{\text {clo }}$.

On observe sur la figure 34 de nouvelles barrières remarquables pour les basses températures de l'air associées à des fortes températures moyennes radiantes. Ce sont des lieux de nouds d'indifférence à $\mathcal{R}_{\text {clo }}$ (en trait épais). Ces ensembles de nouds obtenus pour $\phi$, $A C T$ et $V_{\text {air }}$ variables sont des barrières basses en dessous desquelles les jeux de courbes divergent, l'indépendance du clo est due à l'égalité entre la température moyenne cutanée et la température des

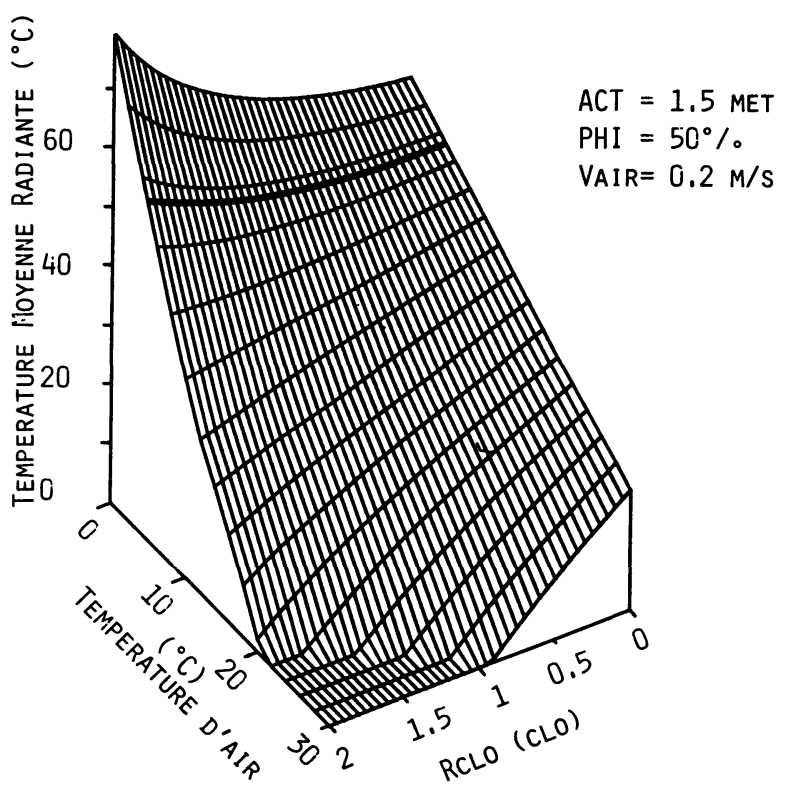

Fig. 34. - Diagramme de confort tridimensionnel (température d'air, température moyenne radiante, isolation thermique du vêtement) pour une activité du sujet de 1,5 Met, une hygrométrie de $50 \%$ et une vitesse relative de l'air de $0,2 \mathrm{~m} / \mathrm{s}$. En trait épais, l'indifférence du vêtement.

[Tridimensional comfort diagram (air temperature, mean radiant temperature, clothing insulation) for a $1.5 \mathrm{Met}$ activity $0.2 \mathrm{~m} / \mathrm{s}$ relative air velocity and $50 \%$ air hygrometry. In thick line, the non influence of clothing insulation.]

Revue de Physioue appliQuée. - T. 19, oo 7, JUILlet 1984 vêtements, dans ce cas, il est aisé de constater que l'on a la double égalité :

$$
T_{\mathrm{sk}}=T_{\mathrm{clo}}=T_{\mathrm{op}}
$$

cette situation peut se rencontrer dans les stations de ski où l'on rencontre une grande variété de vêtements (depuis le maillot de bain qui correspond à $0<\mathcal{R}_{\text {clo }} \lesssim$ 0,3 aux tenues « chaudes " pour lesquelles $2 \leqslant \mathcal{R}_{\text {clo }} \leqslant$ 3 ), la vitesse de l'air étant dans ces cas compensée par l'activité.

4.2.3 L'hygrométrie. - En général, on s'accorde à penser que l'influence de l'hygrométrie est faible dans les climats tempérés, à condition toutefois qu'elle soit dans des limites supportables par le système respiratoire.

Vogt et Miller-Chagas (cités dans [28]) définissent deux limites : une limite inférieure d'humidité correspondant à $10 \mathrm{mmHg}$ de pression partielle de vapeur d'eau. En dessous de cette valeur d'humidité apparaît une sensation de sécheresse des muqueuses buccopharyngiennes. Une limite supérieure fixée à $80 \%$ d'humidité relative au-dessus de laquelle le désagrément est dû aux condensations provoquées à ces niveaux d'humidité. L'influence de l'hygrométrie est principalement le pouvoir évaporateur de l'ambiance, caractérisé par l'écart entre la pression partielle de vapeur d'eau dans l'air et la pression de vapeur saturante au niveau cutané. Un air trop humide ne permet pas une évaporation suffisante de la sueur et dans certains cas, l'énergie excédentaire stockée ne pouvant pas être évacuée normalement, il peut s'en suivre une hyperthermie. Ces problèmes sont beaucoup plus aigus dans les climats tropicaux humides et feront l'objet d'une publication ultérieure. Il n'apparaît pas de nœeud d'indifférence à l'hygrométrie (Fig. 35). Cependant, il est bon de préciser, pour les basses températures d'air, que la perte des qualités thermiques de la majorité des textiles pour les fortes hygrométries, induit le comportement de chauffer plus en métropole par

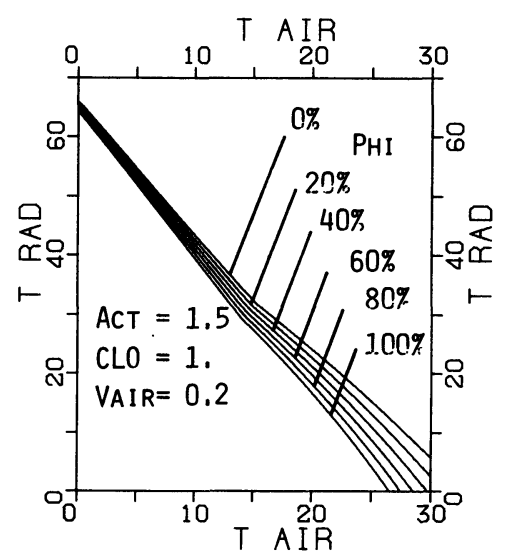

Fig. 35. - Diagramme de confort paramétré en fonction de l'hygrométrie de l'air.

[Comfort diagram with air hygrometry as a parameter.] 
journées humides. Cela n'apparaît pas sur la courbe qui est paramétrée à $\mathcal{R}_{\text {clo }}$ constant et non pas à " vêtement" donné. Par contre, on peut remarquer (en accord avec les courbes obtenues) que l'on tolère mieux un air chaud et sec de type saharien qu'un air moins chaud mais très humide de type maritime tropical ou méditerranéen.

4.2.4 L'activité. - Une augmentation de l'activité conduit directement à une production métabolique plus importante et induit indirectement une augmentation de la vitesse relative de l'air (par déplacement). Dans ces conditions, pour assurer le confort, on se doit d'abaisser la température ambiante (Fig. 36) ou d'abaisser le clo en se découvrant, ceci afin de permettre une évacuation du surcroît d'énergie produite par l'activité métabolique.

$\mathrm{Si}$ les fortes activités, surtout prolongées, sont associées à des températures basses, les influences prédominantes sont obtenues par l'humidité et la vitesse de l'air qui règlent le pouvoir évaporateur de la sueur.
4. 3 LES ZONES DE CONFORT. - Les combinaisons des paramètres qui sont associées à des situations de confort se regroupent en différentes zones à l'intérieur de l'espace défini par les six variables que sont l'activité, l'habillement, l'hygrométrie, la température d'air, la température de rayonnement et la vitesse d'agitation de l'air.

4.3.1 Zone de confort optimal. - Dans la zone bornée par les nœuds d'indifférence sur clo et $V_{\text {air }}$, le passage d'une situation de confort à une autre ou même le positionnement sur une telle situation se fait par thermorégulation (la transpiration variant par exemple de 0 à $30 \%$ de ses possibilités) et par ajustement du métabolisme (l'activité variant inconsciemment de quelques dixièmes de Met). C'est pourquoi il convient de traiter le problème du confort par zones.

On constate que les lignes de confort, qui correspondent aux différents paramétrages des situations de confort, s'enchevêtrent les unes dans les autres dans cette zone. Il y a ainsi passages possibles d'une combinaison de paramètres à une autre avec maintien

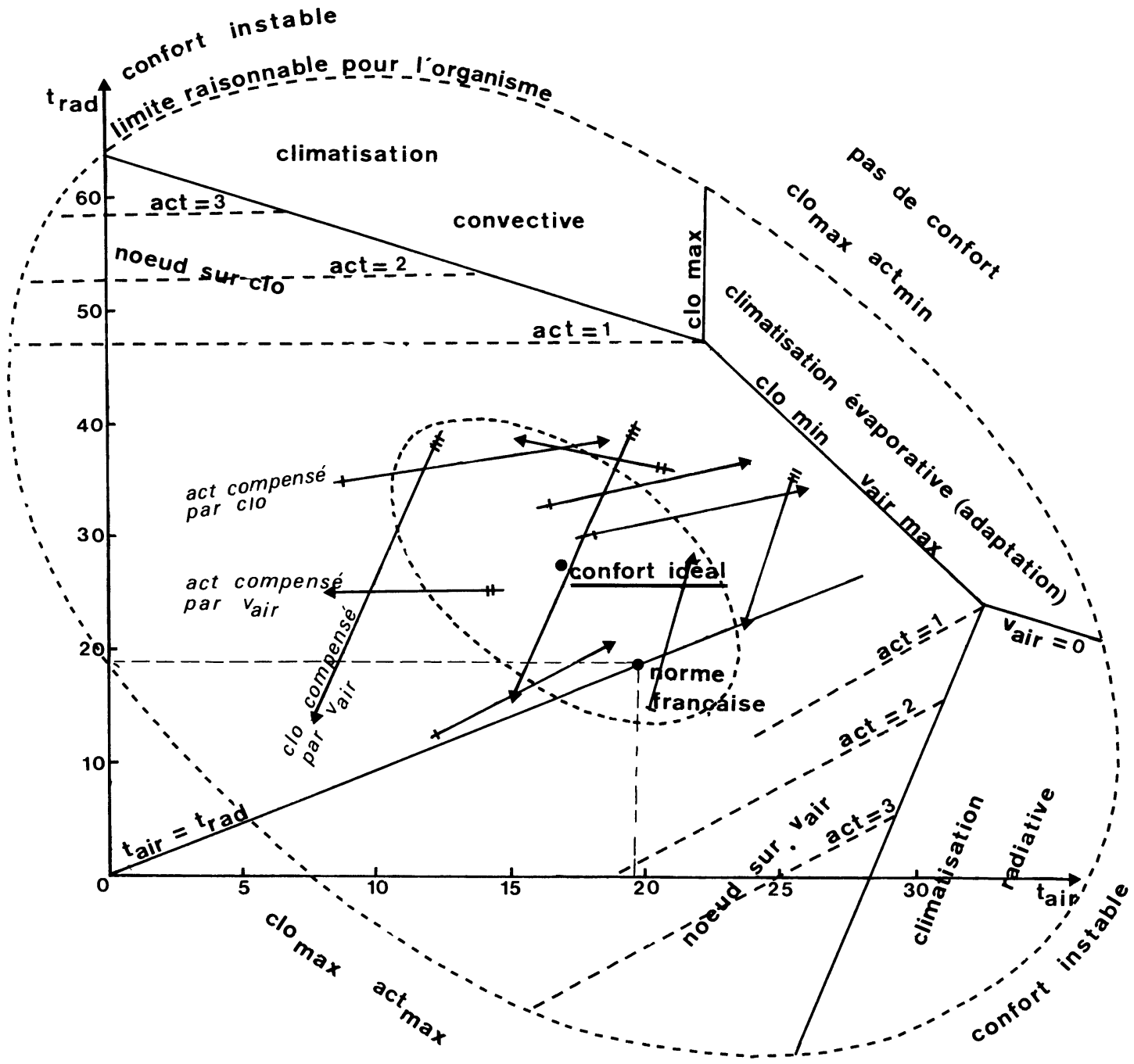

Fig. 36. - Les zones de confort.

[Comfort zones.] 
du confort. Dans ce cas, il y a compensation d'un paramètre par un autre. Dans les cas réels, en effet, contrairement aux expérimentations en chambre bioclimatique, l'être humain modifie consciemment ou non son activité et son habillement quand il ne lui est pas possible d'agir sur les paramètres climatiques.

Mc Intyre [29] fait remarquer que les ajustements concernant l'appareil vestimentaire opérés par l'individu, lui permettent d'élargir la bande de confort jusqu'à $6 \mathrm{~K}$. Toutefois, le régime établi n'étant pas immédiat, le succès de l'ajustement implique une certaine constance dans les températures. Des enquêtes in situ [30] ont montré que de faibles fluctuations des variables climatiques favorisaient les ajustements individuels et donc la satisfaction des usagers au climat intérieur.

L'influence de l'hygrométrie est très faible dans cette zone, en dehors des valeurs extrêmes qui engendrent l'inconfort (voir plus haut).

A une ambiance donnée, définie par une température d'air et une température de rayonnement, peuvent correspondre jusqu'à trois combinaisons distinctes des paramètres $V_{\text {air }}, \mathcal{R}_{\text {clo }}$ et $A C T$ pour une situation de confort.

Les deux zones de nœuds d'indifférence (sur clo et $V_{\text {air }}$ ) qui bornent la zone de confort optimal correspondent à des situations particulières appréciées mais qui, pour des raisons énergétiques, sont rencontrées seulement en extérieur et pendant des durées limitées (l'une de jour, l'autre de nuit).

4.3.2 Zone de climatisation. - Deux zones de climatisation encadrent de part et d'autre les zones de nœuds d'indifférences.

Au-delà des zones de nouds sur $V_{\text {air }}$ on rencontre la climatisation radiative froide et en-deçà de la zone de nouds sur le clo, la climatisation convective.

Ces zones sont à confort instable puisque les lignes de confort divergent les unes des autres : toute variation de l'un des paramètres détruit le confort (il n'y a plus compensation). Ainsi l'être humain doit-il maintenir un même taux d'activité et un même type d'habillement pour assurer son confort dans une ambiance donnée. La mode vestimentaire ne peut pas s'offrir les excentricités qu'elle se permet en climat tempéré. Dans ces zones, l'organisme est en adaptation permanente et l'on fatigue plus ou moins vite. Néanmoins, s'il dispose d'un nombre d'heures suffisant [31] par jour pendant lesquelles il est en situation de confort optimal, l'homme peut supporter sans ennui ces situations de climatisation.

4.3.3 Zones de défense de l'organisme. - Ce sont les deux zones de défense où il n'est plus question de confort : climats chauds et climats froids pour lesquels une isolation du milieu extérieur est indispensable en même temps qu'une adaptation (et une acclimatation) comportementale doit être adoptée : clo élevé et activité forte en climat froid - protection contre le rayonnement (sélectivité) et contrôle de l'évapotranspiration (couche d'air maintenue entre la peau et le vêtement; effet "soufflet "), deux aspects particuliers du vêtement, et activité faible en climat chaud.

Au-delà de ces zones, qui n'ont pas de limites nettes du côté opposé à la zone de confort optimal, il n'y a plus aucun confort possible et l'organisme souffre.

\section{Conclusions.}

La zone de confort optimal est principalement localisée dans l'espace $T_{\mathrm{MR}}>T_{\text {air }}$ ce qui amène quelques remarques :

a) l'être humain est fait pour vivre dans une ambiance radiative : le soleil lui est nécessaire, ainsi que la vie en extérieur : en témoigne la vie actuelle trop sédentaire qui conduit à un attrait indéniable vers les régions ensoleillées;

b) la solution de chauffage par radiateurs, autrefois répandue, et qui a laissé place à celle par convecteurs, était meilleure. Elle engendrait des gradients spatiaux qui agissaient comme stimulants de l'organisme et pas nécessairement comme des inconvénients. Il faut rappeler, en effet, qu'un des critères de confort de l'être humain, est la nécessité d'avoir, toutes proportions gardées bien entendu, des situations de confort modifiées dans le temps pour éviter la saturation des organes de perception. Une directivité adéquate du rayonnement permet des économies d'énergies et évite de créer des zones surchauffées non habitées (hauts de pièces). Par ailleurs, le chauffage radiatif est particulièrement adapté aux variations d'activité de l'être humain : à une augmentation d'activité correspond implicitement une augmentation de la vitesse d'agitation de l'air par suite des mouvements de la personne, ce qui amplifie les déperditions convectives, d'autant plus que l'air est frais, et maintient le confort. Un ralentissement d'activité par contre donne une importance relative plus grande au rayonnement qu'à l'air, ce qui procure le réchauffement nécessaire pour compenser la baisse d'activité.

Le chauffage radiatif est aussi adapté aux allées et venues des personnes puisque les apports calorifiques humains peuvent être compensés immédiatement par une baisse de la température de rayonnement par suite de l'interception effectuée.

La température d'ambiance recommandée en France $\left(19^{\circ} \mathrm{C}\right)$ est en général dans la zone de confort optimal tant que les parois du local considéré ne prennent pas des valeurs anormalement éloignées de la température d'air. Elle a été choisie pour correspondre à des ambiances uniformes air-rayonnement qui ne peuvent être réalisées qu'à certains moments.

L'existence d'apports solaires directs violents, de niveaux d'activité ou d'isolation variables au cours d'un cycle circadien (le sommeil dans un lit correspond à $A C T=0,8$ et $\mathcal{R}_{\text {clo }}=3$ clo alors qu'un travail de bureau correspond généralement à $A C T=1,2$ et 
$\mathcal{R}_{\text {clo }}=1$ clo), et la variété des modes de chauffages existants (poêles, cheminées, panneaux radiants divers, convecteurs, où les parts convectives et radiatives peuvent être très différentes) tendent à limiter l'intérêt pratique de cette recommandation.

Le rôle des échanges rayonnants avec l'organisme mérite d'être réhabilité et permet de rendre compte de situations hors de la normale.

Les matériaux récents et les technologies de contrôle des températures de surface de paroi offrent une possibilité nouvelle de restituer à l'organisme humain une sensation de confort sans pour cela imposer une température aérienne strictement limitée au voisinage de $19^{\circ} \mathrm{C}$.
Il pourrait résulter de leur usage dans le bâtiment des économies d'énergie notables en évitant de chauffer l'air de pieces de grandes hauteurs par exemple) et une plus grande souplesse d'utilisation des locaux (moindre sensibilité à l'ouverture des portes et au passage des personnes).

\section{Remerciements.}

Je tiens à remercier ici Xavier Berger (CNRS) pour l'aide qu'il a apportée à la rédaction de ce document ainsi que Christine Ubaldi pour son excellent travail de secrétariat.

\section{Bibliographie}

[1] American Society of Heating, Refrigerating and Air Conditionning Engineers, " Thermal Comfort conditions ", ASHRAE Stand., New-York (1974) p. 55-74.

[2] FANGER, P. O., "Calculation of thermal comfort : introduction of a basic comfort equation ". ASHRAE Trans. 73, II, 1967.

[3] NisHI, Y.. " Measurement of thermal balance of man ", K. Cena and J. A. Clark eds, Bioengineering, thermal physiology and comfort (Elsevier Scientific Publ. Co., Amsterdam) 1981, p. 29-39.

[4] Du Bors, D. et Du Bors, E. F., Chemical Calorimetry. $\mathrm{X}$. Oma Formula to Estimate the Approximate Surface Area if Height and Weight be known. Arch. Intern. Med. 17 (1916) 863-871.

[5] Fanger, P. O., Angelius, O., KJerulf-Jensen, P., "Radiation Data for the Human Body ", ASHRAE Trans. 76, 2 (1970) 338-373.

[6] Olesen, S., Fanger, P. O., Jensen, P. B. and Nielsen, O. J., «Comfort Limits for Man Exposed to Asymmetric Radiations ", Proc. of the CIB Commission W45 (Human Requirements) Symposium : Thermal Comfort and Moderate Heat Stress, Building Research Station, London, Sept. 1972 (Publ. by HMSO) 1973, p. 133-148.

[7] MC INTYRE, D. A. and GrIFFITHS, I. D., " Radiant Temperature and Comfort ", Proc. of the CIB Commission W45 (Human Requirements) Symposium : Thermal Comfort and Moderate Heat Stress, Building Research Station, London, Sept. 1972 (Publ. by HMSO) 1973, p. 113-132.

[8] Kerslake, D. MC. K., " An Estimate of the Prefered Skin Temperature Distribution in Man ». Ministry of Defense (Air Force Dept.) Flying Personnel Res. Cte. FPRC) Memo 213, 4 pages. Cité par LANDSBERG, H. E., "L'estimation du bioclimat humain ", Revue partielle des paramètres physiques, Technical Note, $\mathrm{n}^{\circ} .123$, W.M.O. (1972) 331.
[9] Deval, J. C. and Berger, X., " Ambiances de confort ", Proc. de la Rencontre de la Société Française des Thermiciens : Les Applications du Thermoconditionnement d̀ la thermique du bâtiment, Lyon 1983, p. $153-169$.

[10] Audoin, P., Berger, X., Pause, P. and Schneider, M., " Etudes d'ambiances de rayonnement ", Cahiers $d u$ C.S.T.B. $\mathrm{n}^{\circ} 218$ (1980).

[11] Nielsen, Pedersen and Lorentz, "Studies on the Heat Loss by Radiation and Convection from the Clothed Human Body ", Acta Physiol. Scand. 27 (1952) 272.

[12] Nielsen, Marins, Pedersen and Lorentz, " Man's Heat Loss by Convection and Radiation ", VARME 18 (1953) 17-27.

[13] Winslow, Gagge and Herrington, "The Influence of air movement upon Heat Losses from the Clothed Human Body », J. Physiol. 127 (1939) 505-518.

[14] Candas, V., Libert, J. P. et Vogt, J. J., « Efficacité évaporatoire de la sudation ", Proc. de la rencontre de la Société Française des Thermiciens : Energétique et thermique de l'homme dans son environnement, Strasbourg 1977, p. D1-D11.

[15] Vogt, J. J., Candas, V., Libert, J. P. and Daull, F., " Required Sweat Rate as an Index of Thermal Strain in Industry ", in K. Cena and J. A. Clark eds, Bioengineering, Thermal Physiology and Comfort (Elsevier Scientific Publ. Co., Amsterdam) 1981, p. 99-110.

[16] Candas, V., Libert, J. P. and Vogt, J. J., Influence of Air Velocity and Heat Acclimation on Human Skin Wettedness and Sweating Efficiency, $J$. Appl. Physiol. 47 (6) (1979) 1194-1200.

[17] Candas, V., Libert, J. P. and Vogt, J. J. : Sweating and Sweat Decline of Resting Men in Hot Humid Environment, Eur. J. Appl. Physiol. 50 (1983) 223-234. 
[18] Vogt, J. J., CANDas, V. and Libert, J. P., « Graphical Determination of Heat Tolerance Limits ", Ergonomics, vol. 25, 4 (1982) 285-294.

[19] Fanger, P. O., "Thermal Comfort " (Mc Graw Hill Book Company, New York) 1973.

[20] MC CUTChAN and TAYLOR, "Respiratory Heat Exchange with varying Temperature and Humidity of Inspired Air ", J. Appl. Physiol. 4 (1951) 121-135.

[21] Meyer, J. P., « Prévision de la température cutanée moyenne : effet des paramètres d'ambiance thermique, de l'exercice musculaire et de l'acclimatement à la chaleur ", Thèse présentée pour le Doctorat en Médecine, Université Louis Pasteur, 1981.

[22] Olesen, B. W. and Fanger, P. O., «The Skin Temperature Distribution for Resting Man in Comfort ", Arch. Sci. Physiol. 27 (4) (1973) A385-A393.

[23] Mitchell, D. and Wyndham, C. H., "Comparison of Weighting Formula for Calculating Mean Skin Temperature ", J. Appl. Physiol. 26 (1969) 616-622.

[24] GAGge, A. P., « Rational Temperature Indices of Thermal Comfort ", in K. Cena and Clark, J. A. (Eds) : Bioengineering, Thermal Physiology and Comfort (Elsevier Scientific Publishing Co., Amsterdam) 1981, 79-98.
[25] Gonzalez, R. R., Nishy, Y. and Gagge, A. P., Rapport du Congrès International de Prague, Microclimats dans l'Industrie (1975) 62-73.

[26] Gagge, A. P., «Standard Operative Temperature, a Generalized Temperature Scale Applicable to Direct and Partitional Calorimetry ", Amer. J. Physiol. 131 (1940) 93-103.

[27] MC INTYRE, D. A., Indoor Climate (Applied Science Publishers Ltd), 1980, 443 pages, 1980.

[28] Vogt, J. J., "Ambiance thermique " (Chap. IV), Organisation Physiologique du Travail, Hygiène, Ergonomie, p. 105-116.

[29] MC INTYRE, D. A., " Design Requirements for a Comfortable Environments ", in Bioengineering, Thermal Physiology and Comfort eds K. Cena and J. A. Clark (Elsevier Scientific Publ. Co., Amsterdam) 1981 , p. 195-220.

[30] Humphrey, M. A., " Field Studies of Thermal Comfort Compared and Applied ", Build. Serv. Eng. 44 (1976) 5-23 and 27.

[31] Metz, B., Etudes Physiologiques des Travailleurs au Sahara ; Proc. des Journées d'Information MédicoSociales Sahariennes; Comité de Coordination Scientifique du Sahara; Maison de l'Unesco, Paris, 1960. 